\title{
Article
}

\section{Rectangular $G_{b}$-Metric Spaces and Some Fixed Point Theorems}

\author{
Chaobo Li (D) and Yunan Cui *D \\ Department of Mathematics, Harbin University of Science and Technology, Harbin 150080, China; \\ lichaobo6898@163.com \\ * Correspondence: cuiya@hrbust.edu.cn
}

Citation: Li, C.; Cui, Y. Rectangular $\mathrm{G}_{b}$-Metric Spaces and Some Fixed Point Theorems. Axioms 2022, 11, 108. https://doi.org/10.3390/

axioms11030108

Academic Editor: Hsien-Chung Wu

Received: 18 January 2022

Accepted: 9 February 2022

Published: 1 March 2022

Publisher's Note: MDPI stays neutral with regard to jurisdictional claims in published maps and institutional affiliations.

Copyright: (C) 2022 by the authors. Licensee MDPI, Basel, Switzerland. This article is an open access article distributed under the terms and conditions of the Creative Commons Attribution (CC BY) license (https:// creativecommons.org/licenses/by/ $4.0 /)$.

\begin{abstract}
In this paper, we first introduce the concept of rectangular $G_{b}$-metric space which generalizes the notion of rectangular metric space and $G_{b}$-metric space. Then, some fixed point results connected with certain contractions are obtained in the setting of rectangular $G_{b}$-metric spaces. Additionally, we also introduce the concept of convex rectangular $G_{b}$-metric space by means of the convex structure and study the fixed points of enriched type contractions in this space.
\end{abstract}

Keywords: $G_{b}$-metric space; rectangular $G_{b}$-metric space; fixed points

MSC: 47H09; 47H10

\section{Introduction}

In recent decades, fixed point theory has had a rapid development. On the one hand, the study of new spaces have been an interesting topic among the mathematical research community. In 1993, Czerwik [1] introduced the concept of $b$-metric space as a generalization of metric space, and generalized Banach contraction principle [2] to this space. Since then, Branciari [3] developed the notion of rectangular metric space via substituting the triangle inequality with the quadrilateral inequality. Recently, George et al. [4] introduced the notion of rectangular $b$-metric space as a generalization of rectangular metric space and they also proved some well-known fixed point results. Meanwhile, Mustafa et al. [5,6] introduced a new class of generalized metric spaces aim to recovered the flaws of Dhage's theory, called $G-$ metric spaces. Later, Aghajani et al. [7,8] generalized the concept of $G$-metric space to $G_{b}$-metric space through $b$-metric and proved some fixed point theorems in this spaces. Many fixed point results in metric spaces see [9-18].

On the other hand, Takahashi [19] introduced a notion of convexity structure in a metric space which offers the minimal tools for constructing various fixed point iterative methods for approximating fixed points of nonlinear operators. Very recently, Chen et al. [20] introduced the notion of convex $b$-metric space, and extend Mann's algorithms directly to $b$-metric spaces. Enriched contraction was introduced by Berinde et al. [21] as follows: Let $(E,\|\cdot\|)$ be a linear normed space. A mapping $T$ is said to be an enriched contraction if

$$
\|k(u-v)+T u-T v\| \leq \theta\|u-v\|, \text { for all } u, v \in E,
$$

where $k \in[0, \infty)$ and $\theta \in[0, k)$. For more results on enriched type contraction, the reader may see [22-25].

In this paper, we aim to extend the concept of $G_{b}$-metric space by defining the notion of rectangular $G_{b}$-metric space which is not necessarily Hausdorff. Then we obtain some well known fixed point theorems such as the Banach contraction principle, the Ćirić type fixed point theorem and the Reich type theorem. Furthermore, we also first introduce the concept of a convex rectangular $G_{b}$-metric space by means of the convex structure and generalize enriched type contraction in this space.

Let us give some basic notations and fundamental results. 


\section{Preliminaries}

Definition 1 ([1]). Let $E$ be a nonempty set, and for all $u, v, z \in E$, let a function $d: E \times E \rightarrow$ $[0, \infty)$ satisfies the following conditions:

(1) $d(u, v)=0$ if and only if $u=v$;

(2) $d(u, v)=d(v, u)$;

(3) there exists a real number $s \geq 1$ such that $d(u, v) \leq s[d(u, z)+d(z, v)]$.

Then $d$ is called $a b$-metric on $E$ and the pair $(E, d)$ is called a b-metric space (bMS) with coefficient $s \geq 1$.

Definition 2 ([3]). Let $E$ be a nonempty set, and for all $u, v \in E$, let a function $d: E \times E \rightarrow[0, \infty)$ satisfies the following conditions:

(1) $d(u, v)=0$ if and only if $u=v$;

(2) $d(u, v)=d(v, u)$;

(3) $d(u, v) \leq d(u, z)+d(z, h)+d(h, v)$ for all distinct points $z, h \in E \backslash\{u, v\}$.

Then $d$ is called a rectangular metric on $E$ and $(E, d)$ is called a rectangular metric space (RMS).

Definition 3 ([4]). Let $E$ be a nonempty set, and for all $u, v \in E$, let a function $d: E \times E \rightarrow[0, \infty)$ satisfies the following conditions:

(1) $d(u, v)=0$ if and only if $u=v$;

(2) $d(u, v)=d(v, u)$;

(3) there exists a real number $s \geq 1$ such that $d(u, v) \leq s[d(u, z)+d(z, h)+d(h, v)]$ for all distinct points $z, h \in E \backslash\{u, v\}$.

Then $d$ is called a rectangular b-metric on $E$ and $(E, d)$ is called a rectangular b-metric space $\left(R_{b} M S\right)$ with coefficient $s \geq 1$.

Example 1 ([4]). Let $E=\Gamma \cup \mathrm{H}$, where $\Gamma=\left\{\frac{1}{n}: n \in \mathbb{N}\right\}$ and $H$ is the set of all positive integers. Let $d_{b}: E \times E \rightarrow[0, \infty)$ such that $d_{b}(u, v)=d_{b}(v, u)$ for all $u, v \in E$ and

$$
d_{b}(u, v)= \begin{cases}0, & \text { if } u=v ; \\ 2 a, & \text { if } u, v \in \Gamma \\ \frac{a}{2 n}, & \text { if } u \in \Gamma \text { and } v \in\{2,3\} \\ a, & \text { otherwise, }\end{cases}
$$

where $a>0$ is a constant. Then $\left(E, d_{b}\right)$ is $a R_{b} M S$ with coefficient $s=2>1$.

Definition 4 ([5]). Let $E$ be a nonempty and for all $u, v, o \in E$, let a function $G: E \times E \times E \rightarrow$ $[0, \infty)$ satisfies the following conditions:

(1) $G(u, v, o)=0$ if $u=v=o$;

(2) $0<G(u, u, v)$ with $u \neq v$;

(3) $G(u, u, v) \leq G(u, v, z)$ for all $z \in E$ with $v \neq z$;

(4) $G(u, v, o)=G(u, o, v)=G(v, o, u)=\ldots$, (symmetry in all three variables);

(5) $G(u, v, o) \leq G(u, z, z)+G(z, v, o)$ for all $z \in E$.

Then $G$ is called $a G$-metric on $E$ and the pair $(E, G)$ is called a $G$-metric space (GMS).

Example 2 ([6]). Let $E=\mathbb{R} \backslash\{0\}$ and define $G: E \times E \times E \longrightarrow[0, \infty)$ by

$$
G(u, v, o)=\left\{\begin{array}{l}
1+|u-v|+|v-o|+|u-o| \text { if } u, v, o \text { all have the same sign; } \\
|u-v|+|v-o|+|u-o| \text { otherwise, }
\end{array}\right.
$$

for all $u, v, o \in E$. Then $(E, G)$ is a GMS.

Definition 5 ([7]). Let $E$ be a nonempty and for all $u, v, o \in E$, let a function $G: E \times E \times E \rightarrow$ $[0, \infty)$ satisfies the following conditions: 
(1) $G(u, v, o)=0$ if $u=v=o$;

(2) $0<G(u, u, v)$ with $u \neq v$;

(3) $G(u, u, v) \leq G(u, v, z)$ for all $z \in E$ with $v \neq z$;

(4) $G(u, v, o)=G(u, o, v)=G(v, o, u)=\ldots$, (symmetry in all three variables);

(5) there exists a real number $s \geq 1$ such that $G(u, v, o) \leq s[G(u, z, z)+G(z, v, o)]$.

Then $G$ is called $a G_{b}$-metric on $E$ and the pair $(E, G)$ is called $a G_{b}$-metric space $\left(G_{b} M S\right)$.

Example 3 ([7]). Let $(E, G)$ be a GMS and $G_{b}$-metric defined by $G_{b}(u, v, o)=(G(u, v, o))^{p}$. Then $\left(E, G_{b}\right)$ is a $G_{b} M S$ with $s=2^{p-1}$.

Each $G M S$ is a $G_{b} M S$ with $s=1$, and a $G_{b} M S$ is not necessarily a GMS (see [7]).

Definition 6 ([5]). A $G$-metric is said to be symmetric if $G(u, v, v)=G(v, u, u)$ for all $u, v \in E$.

\section{Rectangular $G_{b}$-Metric Spaces}

Now, we define the notion of rectangular $G_{b}$-metric spaces.

Definition 7. Let $E$ be a nonempty and for all $u, v, o \in E$, let a function $G: E \times E \times E \rightarrow[0, \infty)$ satisfies the following conditions:

(RGb1) $G(u, v, o)=0$ if $u=v=o$;

$(R G b 2) 0<G(u, u, v)$ with $u \neq v$;

(RGb3) $G(u, u, v) \leq G(u, v, z)$ for all $z \in E$ with $v \neq z$;

$(R G b 4) G(u, v, o)=G(u, o, v)=G(v, o, u)=\ldots$, (symmetry in all three variables);

(RGb5) there exists a real number $s \geq 1$ such that $G(u, v, o) \leq s[G(u, z, z)+G(z, h, h)+$

$G(h, v, o)]$ for all distinct points $z, h \in E \backslash\{u, v\}$.

Then $G$ is called a rectangular $G_{b}$-metric on $E$ and the pair $(E, G)$ is called a rectangular $G_{b}$-metric space $\left(R G_{b} M S\right)$.

\section{Remark 1.}

(1) It is worth mentioning that $R G_{b} M S$ generalizes rectangular $G$-metric space (RGMS), because a $R G_{b} M S$ reduces a $R G M S$ for $s=1$.

(2) Every $G_{b} M S$ with coefficient $s$ is a $R G_{b} M S$ with coefficient $s^{2}$. Indeed, let $\left(E, G_{b}\right)$ is a $G_{b} M S$, for all distinct $z, h \in E \backslash\{u, v, o\}$, we have

$$
\begin{aligned}
G_{b}(u, v, o) & \leq s\left[G_{b}(u, z, z)+G_{b}(z, v, o)\right] \\
& \leq s\left[G_{b}(u, z, z)+s G_{b}(z, h, h)+s G_{b}(h, v, o)\right] \\
& \leq s^{2}\left[G_{b}(u, z, z)+G_{b}(z, h, h)+G_{b}(h, v, o)\right]
\end{aligned}
$$

which shows that $\left(E, G_{b}\right)$ is a $R G_{b} M S$ with coefficient $s^{2}$.

Let us present some specific examples of $R G_{b} M S$.

Example 4. Let $\left(E, G_{*}\right)$ be a $G$-metric space. Define $G: E \times E \times E \rightarrow[0, \infty)$ by $G(u, v, o)=$ $\left[G_{*}(u, v, o)\right]^{r}$ for all $u, v, o \in E$ and $r \geq 1$. Then, $(E, G)$ is a $R G_{b} M S$ with $s=3^{r-1}$. Obviously, $G$ satisfies conditions $(R G b 1)-(R G b 4)$ of the Definition 7. Next, we shall prove that (RGb5) is hold. If $1 \leq r<\infty$, from the convexity of function $f(u)=u^{r}$ for $u \geq 0$, we have

$$
(a+b+c)^{r} \leq 3^{r-1}\left(a^{r}+b^{r}+c^{r}\right),
$$


for nonnegative real numbers $a, b, c$. Thus for distinct points $z, h \in E \backslash\{u, v, o\}$, we obtain

$$
\begin{aligned}
G(u, v, o) & =\left[G_{*}(u, v, o)\right]^{r} \\
& \leq\left[G_{*}(u, z, z)+G_{*}(z, h, h)+G_{*}(h, v, o)\right]^{r} \\
& \leq 3^{p-1}\left(\left[G_{*}(u, z, z)^{r}+G_{*}(z, h, h)^{r}+G_{*}(h, v, o)^{r}\right]\right. \\
& =3^{r-1}[G(u, z, z)+G(z, h, h)+G(h, v, o)] .
\end{aligned}
$$

Therefore, $(E, G)$ is a $R G_{b} M S$ with $s=3^{r-1}$.

Example 5. Let $E=\mathbb{R}$ and we define $G: E \times E \times E \rightarrow[0, \infty)$ by the formula

$$
G(u, v, o)=(|u-v|+|v-o|+|u-o|)^{r},(r \geq 1)
$$

Then $(E, G)$ is a $R G_{b} M S$ with $s=3^{r-1}$. Obviously, $G$ satisfies conditions (RGb1) - (RGb4) of the Definition 7. Next, we shall verify that $G$ satisfies (RGb5). If $1 \leq r<\infty$, from the convexity of function $f(u)=u^{r}$ for $u \geq 0$, we have

$$
(a+b+c)^{r} \leq 3^{r-1}\left(a^{r}+b^{r}+c^{r}\right),
$$

for nonnegative real numbers $a, b, c$. Thus for distinct points $z, h \in E \backslash\{u, v, o\}$, we obtain

$$
\begin{aligned}
G(u, v, o) & =(|u-v|+|v-o|+|u-o|)^{r} \\
& \leq(|u-z|+|z-v|+|v-o|+|u-z|+|z-o|)^{r} \\
& \leq(|u-z|+|z-h|+|h-v|+|v-o|+|u-z|+|z-h|+|h-o|)^{r} \\
& =[(|u-z|+|u-z|)+(|z-h|+|z-h|)+(|h-v|+|v-o|+|h-o|)]^{r} \\
& \leq 3^{r-1}\left[(|u-z|+|u-z|)^{r}+(|z-h|+|z-h|)^{r}+(|h-v|+|v-o|+|h-o|)^{r}\right] \\
& =3^{r-1}[G(u, z, z)+G(z, h, h)+G(h, v, o)] .
\end{aligned}
$$

Hence, $(E, G)$ is a $R G_{b} M S$ with $s=3^{r-1}$.

The following example shows that a $R G_{b} M S$ does not a $R G M S$ and a $G_{b} M S$.

Example 6. Let $E=\Gamma \cup\{1,2\} \cup H$, where $\Gamma=\left\{\frac{1}{n}: n=2,3,4, \ldots\right\}$ and $H=\{3,4,5, \ldots\}$. Define $G: E \times E \times E \rightarrow[0, \infty)$ such that $G(u, v, o)=G(u, o, v)=G(v, o, u)=\ldots$ for all $u, v, o \in E$ and

$$
G(u, v, o)= \begin{cases}0 & \text { if } u=v=o \\ 4 a & \text { if } u, v, o \in \Gamma \text { and } u \neq v \neq o \\ \frac{a}{2 n} \quad \text { if } u \in \Gamma \text { and } v=o \in\{1,2\} \\ a \quad \text { otherwise, }\end{cases}
$$

where $a>0$ is a constant. Then $(E, G)$ is a $R G_{b} M S$ with coefficient $s=2>1$. However, $(E, G)$ is not a RGMS, as $G\left(\frac{1}{2}, \frac{1}{3}, \frac{1}{4}\right)=4 a>\frac{9 a}{4}=G\left(\frac{1}{2}, 1,1\right)+G(1,2,2)+G\left(2, \frac{1}{3}, \frac{1}{4}\right)$ and not $a G_{b} M S$, as there does not exists $s \geq 1$ such that $G(u, v, o) \leq s[G(u, z, z)+G(z, v, o)]$ for all $u, v, o, z \in E$.

Proposition 1. Let $(E, G)$ be a $R G_{b} M S$ and $\varepsilon>0$, then for each $u, v, o \in E$, we have:

(1) if $G(u, v, o)=0$, then $u=v=o$;

(2) if $G\left(u_{n}, u_{m}, u_{l}\right)<\varepsilon$ for any $n, m, l \in \mathbb{N}$, then $G\left(u_{n}, u_{m}, u_{m}\right)<\varepsilon$ and $G\left(u_{n}, u_{n}, u_{m}\right)<\varepsilon$.

\section{Proof.}

(1) By (RGb2) and (RGb3), we get a contradiction. Indeed,

$$
0=G(u, v, o) \geq G(u, u, v)>0,
$$


when $u \neq v$ and $v \neq o$. In addition,

$$
0=G(u, v, o)=G(v, v, o)>0
$$

when $u=v$ and $v \neq o$.

(2) It can be obtain from (RGb3) of the Definition 7.

Proposition 2. Let $(E, G)$ be a $R G_{b} M S$. Then, the function given as $d_{G}(u, v)=G(u, v, v)+$ $G(v, u, u)$, defines a rectangular $b$-metric on $E$. We call it a rectangular $b$-metric $d_{G}$ induced by the rectangular $G_{b}$-metric $G$.

Proof. Let us prove that conditions of Definition 3 are fulfilled for $d_{G}(u, v)$.

(1) If $d_{G}(u, v)=0$, then $G(u, v, v)=G(v, u, u)=0$ and by Proposition 1, it follows that $u=v$. If $u=v$, then $G(u, v, v)=G(v, u, u)=0$ and $d_{G}(u, v)=0$;

(2) Property (RGb4) of the Definition 7 implies that

$$
d_{G}(u, v)=G(u, v, v)+G(v, u, u)=G(v, u, u)+G(u, v, v)=d_{G}(v, u) ;
$$

(3) By (RGb5) of the Definition 7, for distinct points $z, h \in E \backslash\{u, v\}$, it follows that

$$
\begin{aligned}
d_{G}(u, v) & =G(u, v, v)+G(u, u, v) \\
& \leq s[G(u, z, z)+G(z, h, h)+G(h, v, v)]+s[G(v, h, h)+G(h, z, z)+G(z, u, u)] \\
& =s[G(u, z, z)+G(z, u, u)+G(z, h, h)+G(h, z, z)+G(h, v, v)+G(v, h, h)] \\
& =s\left[d_{G}(u, z)+d_{G}(z, h)+d_{G}(h, v)\right] .
\end{aligned}
$$

We now define $G$-convergent and $G-$ Cauchy sequence in $R G_{b} M S$ and completeness of $R G_{b} M S$.

Definition 8. Let $(E, G)$ be a $R G_{b} M S$. The sequence $\left\{u_{n}\right\}$ in $E$ and $u \in E$, then

(1) the sequence $\left\{u_{n}\right\}$ is said to be $G$-convergent in $E$ to $u$, if for any $\varepsilon>0$, there exists a positive integer $n_{0} \in \mathbb{N}$ such that $G\left(u_{n}, u_{m}, u\right)<\varepsilon$ for all $m, n \geq n_{0}$, and this fact is represented by $\lim _{n, m \rightarrow \infty} G\left(u_{n}, u_{m}, u\right)=0$;

(2) the sequence $\left\{u_{n}\right\}$ is said to be $G$-Cauchy sequence in $E$, if for any $\varepsilon>0$, there exists a positive integer $n_{0} \in \mathbb{N}$ such that $G\left(u_{n}, u_{m}, u_{l}\right)<\varepsilon$ for all $n, m, l \geq n_{0}$, and this fact is represented by $\lim _{n, m, l \rightarrow \infty} G\left(u_{n}, u_{m}, u_{l}\right)=0$;

(3) $(E, G)$ is said to be a complete $R G_{b} M S$, if every $G$-Cauchy sequence in $E$ converges to some $u \in E$.

Definition 9. A rectangular $G_{b}$-metric $G$ is said to be symmetric if $G(u, v, v)=G(u, u, v)$ for all $u, v \in E$.

Remark 2. It follows Example 4 that rectangular $G_{b}$-metric $G$ is symmetric if $G-$ metric $G_{*}$ is symmetric.

Definition 10. Let $(E, G)$ be a $R G_{b} M S$, for $u_{0} \in E, r>0$, then $G-$ ball with center $u_{0}$ and radius $r$ is

$$
B_{G}\left(u_{0}, r\right)=\left\{v \in E: G\left(u_{0}, v, v\right)<r\right\} .
$$

Remark 3. Open balls in $R G_{b} M S$ are not necessarily open. In Example $6, B_{G}\left(\frac{1}{2}, \frac{a}{2}\right)=\left\{\frac{1}{2}, 1,2\right\}$ and there does not exists any open ball with center 1 and contained in $B_{G}\left(\frac{1}{2}, \frac{a}{2}\right)$. So $B_{G}\left(\frac{1}{2}, \frac{a}{2}\right)$ is not an open set. 
Proposition 3. Let $(E, G)$ be a $R G_{b} M S$, then for any $u_{0} \in E, r>0$, we have

(1) if $G\left(u_{0}, v, o\right)<r$, then $v, o \in B_{G}\left(u_{0}, r\right)$.

(2) if $G$ is symmetric, then

$$
B_{G}\left(u_{0}, \frac{r}{2}\right) \subseteq B_{d_{G}}\left(u_{0}, r\right) \subseteq B_{G}\left(u_{0}, r\right)
$$

Thus, if rectangular $G_{b}$-metric is symmetric, then the $R G_{b} M S$ is topologically equivalent to a $R_{b} M S$. This allows us to readily transport many concepts and results from $R_{b} M S$ into $R G_{b} M S$ setting.

It should be noted that $R G_{b} M S$ is not necessarily Hausdorff.

Example 7. Let $\left(E, d_{b}\right)$ is a $R_{b} M S$ with $s=2$ as shows in Example 1. For all $u, v, o \in E$, we define $G: E \times E \times E \rightarrow[0, \infty)$ by the formula $G(u, v, o)=\max \left\{d_{b}(u, v), d_{b}(u, o), d_{b}(o, u)\right\}$. Obviously, G satisfies conditions (RGb1) - (RGb4) of the Definition 7, so we only need to verify that (RGb5) is hold. Indeed,

(1) if $u \neq v \neq o$, then $G(u, v, o)=\max \left\{d_{b}(u, v), d_{b}(u, o), d_{b}(o, u)\right\}$;

(2) if $u=v \neq o$, then $G(u, v, o)=G(u, u, o)=d_{b}(u, o)$.

For distinct points $z, h \in E \backslash\{u, v\}$, we have

$$
\begin{aligned}
G(u, v, o) & \leq \max \left\{s\left[d_{b}(u, z)+d_{b}(z, h)+d_{b}(h, v)\right], d_{b}(v, o), s\left[d_{b}(o, h)+d_{b}(h, z)+d_{b}(z, u)\right]\right\} \\
& \leq s\left[d_{b}(u, z)+d_{b}(z, h)\right]+s \max \left\{d_{b}(h, v), d_{b}(v, o), d_{b}(o, h)\right\} \\
& =s[G(u, z, z)+G(z, h, h)+G(h, v, o)] .
\end{aligned}
$$

Hence, $(E, G)$ is a $R G_{b} M S$ with $s=2$. However, there does not exists any $r_{1}, r_{2}>0$ such that $B_{G}\left(2, r_{1}\right) \cap B_{G}\left(3, r_{2}\right)=\varnothing$, thus $(E, G)$ is not Hausdorff.

\section{Fixed Point Results}

In this section, we show some results of fixed points for several contraction mappings in a $R G_{b} M S$ under suitable hypotheses.

Lemma 1 ([26]). Let $(E, G)$ be a $R_{b} M S$ with coefficient $s \geq 1$, and $\left\{u_{n}\right\}$ be a sequence in $E$ such that

$$
d\left(u_{n+1}, u_{n}\right) \leq \lambda d\left(u_{n}, u_{n-1}\right)
$$

for all $n \in \mathbb{N}$ and $\lambda \in[0,1)$. Then sequence $\left\{u_{n}\right\}$ is Cauchy sequence in $E$.

Lemma 2. Let $(E, G)$ be a $R G_{b} M S$ with coefficient $s \geq 1$. Suppose that there exists a symmetric rectangular $G_{b}$-metric $G$ on $E$ and $\left\{u_{n}\right\}$ is a sequence in $E$ induced by $u_{n+1}=T u_{n}$ such that

$$
G\left(u_{n}, u_{n+1}, u_{n+1}\right)+G\left(u_{n}, u_{n}, u_{n+1}\right) \leq \lambda\left(G\left(u_{n-1}, u_{n}, u_{n}\right)+G\left(u_{n-1}, u_{n-1}, u_{n}\right)\right),
$$

for all $n \in N$ and $\lambda \in[0,1)$. Then $\left\{u_{n}\right\}$ is a $G$-Cauchy sequence in $E$.

Proof. The condition in Equation (1), together with Proposition 2, implies that

$$
d_{G}\left(u_{n}, u_{n+1}\right) \leq \lambda d_{G}\left(u_{n-1}, u_{n}\right)
$$

for all $n \in \mathbb{N}$. Utilizing Lemma 1 , it shows that $\left\{u_{n}\right\}$ is a Cauchy sequence in $R_{b} M S$. Since $\left(E, d_{G}\right)$ and $(E, G)$ are topologically equivalent while rectangular $G_{b}$-metric is symmetric, we have that $\left\{u_{n}\right\}$ is also a $G-$ Cauchy sequence in $R G_{b} M S$. 
Lemma 3. Let $(E, G)$ be a $R G_{b} M S$ with coefficient $s \geq 1$ and $\left\{u_{n}\right\}$ be a sequence in $E$ induced by $u_{n+1}=T u_{n}$ such that

$$
G\left(u_{n}, u_{n+1}, u_{n+2}\right) \leq \lambda G\left(u_{n-1}, u_{n}, u_{n+1}\right)
$$

where $\lambda \in[0,1)$ and $n \in \mathbb{N}$. Then $\left\{u_{n}\right\}$ is a $G$-Cauchy sequence in $E$.

Proof. Without loss of generality, we assume that $u_{n} \neq u_{m}$ for all $n \neq m$. By the inequality (2), we obtain

$$
G\left(u_{n}, u_{n+1}, u_{n+2}\right) \leq \lambda^{n} G\left(u_{0}, u_{1}, u_{2}\right)
$$

and

$$
\begin{array}{r}
G\left(u_{n}, u_{n+1}, u_{n+1}\right) \leq \lambda^{n} G\left(u_{0}, u_{1}, u_{2}\right) \\
G\left(u_{n}, u_{n}, u_{n+1}\right) \leq \lambda^{n} G\left(u_{0}, u_{1}, u_{2}\right) .
\end{array}
$$

Setting $G\left(u_{n}, u_{n+1}, u_{n+1}\right)=G_{n}, G\left(u_{n+1}, u_{n}, u_{n}\right)=G_{n}^{\prime}$ and $G\left(u_{n}, u_{n+1}, u_{n+2}\right)=G_{n}^{*}$ for all $n \in \mathbb{N}$. Obviously, $G_{n} \leq G_{n}^{*}$ and $G_{n}^{\prime} \leq G_{n}^{*}$ for all $n \in \mathbb{N}$. In order to prove our conclusion, we divide the proof into three cases.

Case 1 Let $\lambda \in\left[0, \frac{1}{s}\right)(s>1)$. For the sequence $\left\{u_{n}\right\}$, we consider $G\left(u_{n}, u_{n+p}, u_{n+p+q}\right)$ in four subcases.

Subcase 1 If $p$ is even say $2 m(m \geq 1)$, and $q$ is even say $2 l(l \geq 1)$. From Definition 7 , it follows that

$$
\begin{aligned}
& G\left(u_{n}, u_{n+2 m}, u_{n+2 m+2 l}\right) \\
= & G\left(u_{n+2 m+2 l}, u_{n+2 m}, u_{n}\right) \\
\leq & s\left[G\left(u_{n+2 m+2 l}, u_{n+2 m+2 l-1}, u_{n+2 m+2 l-1}\right)+G\left(u_{n+2 m+2 l-1}, u_{n+2 m-1}, u_{n+2 m-1}\right)\right. \\
& \left.+G\left(u_{n+2 m-1}, u_{n+2 m}, u_{n}\right)\right] \\
\leq & s\left[G^{\prime}{ }_{n+2 m+2 l-1}+G\left(u_{n+2 m+2 l-1}, u_{n+2 m+2 l-2}, u_{n+2 m-1}\right)+G\left(u_{n+2 m-1}, u_{n+2 m}, u_{n}\right)\right] \\
= & s G\left(u_{n+2 m-1}, u_{n+2 m+2 l-2}, u_{n+2 m+2 l-1}\right)+s G_{n+2 m+2 l-1}^{\prime}+s G\left(u_{n}, u_{n+2 m-1}, u_{n+2 m}\right) \\
\leq & s^{2}\left[G_{n+2 m-1}+G_{n+2 m}+G\left(u_{n+2 m+1}, u_{n+2 m+2 l-2}, u_{n+2 m+2 l-1}\right)\right]+s G^{\prime}{ }_{n+2 m+2 l-1} \\
& +s G\left(u_{n}, u_{n+2 m-1}, u_{n+2 m}\right) \\
\leq & s^{2}\left[G_{n+2 m-1}+G_{n+2 m}\right]+s^{3}\left[G_{n+2 m+1}+G_{n+2 m+2}\right]+\cdots+s^{l}\left[G_{n+2 m+2 l-5}+G_{n+2 m+2 l-4}\right. \\
& \left.\left.+G_{n+2 m+2 l-3}^{*}\right]+s G_{n+2 m+2 l-1}^{\prime}+s G_{n+2}, u_{n+2 m-1}, u_{n+2 m}\right) \\
\leq & s^{2}\left[\lambda^{n+2 m-1} G_{0}^{*}+\lambda^{n+2 m} G_{0}^{*}\right]+s^{3}\left[\lambda^{n+2 m+1} G_{0}^{*}+\lambda^{n+2 m+2} G_{0}^{*}\right]+\cdots+s^{l}\left[\lambda^{n+2 m+2 l-5} G_{0}^{*}\right. \\
& \left.+\lambda^{n+2 m+2 l-4} G_{0}^{*}+\lambda^{n+2 m+2 l-3} G_{0}^{*}\right]+s \lambda^{n+2 m+2 l-1} G_{0}^{*}+s G\left(u_{n}, u_{n+2 m-1}, u_{n+2 m}\right) \\
\leq & s^{2} \lambda^{n+2 m-1}\left[1+s \lambda^{2}+s^{2} \lambda^{4}+\cdots\right] G_{0}^{*}+s^{2} \lambda^{n+2 m}\left[1+s \lambda^{2}+s^{2} \lambda^{4}+\cdots\right] G_{0}^{*} \\
& +s \lambda^{n+2 m+2 l-1} G_{0}^{*}+s G\left(u_{n}, u_{n+2 m-1}, u_{n+2 m}\right) \\
& 1+\lambda \\
& 1-s \lambda^{2} s^{2} \lambda^{n+2 m-1} G_{0}^{*}+s \lambda^{n+2 m+2 l-1} G_{0}^{*}+s G\left(u_{n}, u_{n+2 m-1}, u_{n+2 m}\right)
\end{aligned}
$$


and

$$
\begin{aligned}
& G\left(u_{n}, u_{n+2 m-1}, u_{n+2 m}\right) \\
\leq & s\left[G\left(u_{n}, u_{n+1}, u_{n+1}\right)+G\left(u_{n+1}, u_{n+2}, u_{n+2}\right)+G\left(u_{n+2}, u_{n+2 m-1}, u_{n+2 m}\right)\right] \\
\leq & s\left[G_{n}+G_{n+1}\right]+s^{2}\left[G_{n+2}+G_{n+3}\right]+\ldots+s^{m-1}\left[G_{n+2 m-4}+G_{n+2 m-3}+G_{n+2 m-2}^{*}\right] \\
\leq & s\left[\lambda^{n} G_{0}^{*}+\lambda^{n+1} G_{0}^{*}\right]+s^{2}\left[\lambda^{n+2} G_{0}^{*}+\lambda^{n+3} G_{0}^{*}\right]+\ldots+s^{m-1}\left[\lambda^{n+2 m-3} G_{0}^{*}+\lambda^{n+2 m-2} G_{0}^{*}\right. \\
& \left.+\lambda^{n+2 m-1} G_{n+2 m-2}^{*}\right] \\
\leq & s \lambda^{n}\left(1+s \lambda^{2}+s^{2} \lambda^{4}+\ldots\right) G_{0}^{*}+s \lambda^{n+1}\left(1+s \lambda^{2}+s^{2} \lambda^{4}+\ldots\right) G_{0}^{*} \\
\leq & \frac{1+\lambda}{1-s \lambda^{2}} s \lambda^{n} G_{0}^{*} .
\end{aligned}
$$

Therefore, we have

$$
G\left(u_{n}, u_{n+2 m}, u_{n+2 m+2 l}\right) \leq \frac{1+\lambda}{1-s \lambda^{2}} s^{2} \lambda^{n+2 m-1} G_{0}^{*}+s \lambda^{n+2 m+2 l-1} G_{0}^{*}+\frac{1+\lambda}{1-s \lambda^{2}} s^{2} \lambda^{n} G_{0}^{*} .
$$

Since $\lambda<\frac{1}{s}$, we can deduce that

$$
G\left(u_{n}, u_{n+2 m}, u_{n+2 m+2 l}\right) \leq \frac{3+\lambda}{1-s \lambda^{2}} s^{2} \lambda^{n} G_{0}^{*}
$$

Subcase 2 If $p$ is even say $2 m(m \geq 1)$, and $q$ is odd say $2 l+1(l \geq 0)$. From Definition 7 , it follows that

$$
\begin{aligned}
& G\left(u_{n}, u_{n+2 m}, u_{n+2 m+2 l+1}\right) \\
&= G\left(u_{n+2 m+2 l+1}, u_{n+2 m}, u_{n}\right) \\
& \leq s\left[G\left(u_{n+2 m+2 l+1}, u_{n+2 m+2 l+2}, x_{n+2 m+2 l+2}\right)+G\left(u_{n+2 m+2 l+2}, u_{n+2 m-1}, u_{n+2 m-1}\right)\right. \\
&\left.+G\left(u_{n+2 m-1}, u_{n+2 m}, u_{n}\right)\right] \\
& \leq s\left[G_{n+2 m+2 l+1}+G\left(u_{n+2 m+2 l+2}, u_{n+2 m+2 l+3}, u_{n+2 m-1}\right)+G\left(u_{n+2 m-1}, u_{n+2 m}, u_{n}\right)\right] \\
&= s G\left(u_{n+2 m-1}, u_{n+2 m+2 l+2}, u_{n+2 m+2 l+3}\right)+s G_{n+2 m+2 l+1}+s G\left(u_{n}, u_{n+2 m-1}, u_{n+2 m}\right) \\
& \leq s^{2}\left[G_{n+2 m-1}+G_{n+2 m}+G\left(u_{n+2 m+1}, u_{n+2 m+2 l+2}, u_{n+2 m+2 l+3}\right)\right]+s G_{n+2 m+2 l+1} \\
&+s G\left(u_{n}, u_{n+2 m-1}, u_{n+2 m}\right) \\
& \leq s^{2}\left[G_{n+2 m-1}+G_{n+2 m}\right]+s^{3}\left[G_{n+2 m+1}+G_{n+2 m+2}\right]+\cdots+s^{l+2}\left[G_{n+2 m+2 l-1}+G_{n+2 m+2 l}\right. \\
&\left.+G_{n+2 m+2 l+1}^{*}\right]+s G_{n+2 m+2 l+1}+s G\left(u_{n}, u_{n+2 m-1}, u_{n+2 m}\right) \\
& \leq s^{2}\left[\lambda^{n+2 m-1} G_{0}^{*}+\lambda^{n+2 m} G_{0}^{*}\right]+s^{3}\left[\lambda^{n+2 m+1} G_{0}^{*}+\lambda^{n+2 m+2} G_{0}^{*}\right]+\cdots+s^{l+2}\left[\lambda^{n+2 m+2 l-1} G_{0}^{*}\right. \\
&\left.+\lambda^{n+2 m+2 l} G_{0}^{*}+\lambda^{n+2 m+2 l-3} G_{0}^{*}\right]+s \lambda^{n+2 m+2 l+1} G_{0}^{*}+s G_{n}\left(u_{n}, u_{n+2 m-1}, u_{n+2 m}\right) \\
& \leq s^{2} \lambda^{n+2 m-1}\left[1+s \lambda^{2}+s^{2} \lambda^{4}+\cdots\right] G_{0}^{*}+s^{2} \lambda^{n+2 m}\left[1+s \lambda^{2}+s^{2} \lambda^{4}+\cdots\right] G_{0}^{*} \\
&+s \lambda^{n+2 m+2 l+1} G_{0}^{*}+s G_{n}\left(u_{n}, u_{n+2 m-1}, u_{n+2 m}\right) \\
&= 1+\lambda \\
& 1-s \lambda^{2} s^{2} \lambda^{n+2 m-1} G_{0}^{*}+s \lambda^{n+2 m+2 l+1} G_{0}^{*}+s G\left(u_{n}, u_{n+2 m-1}, u_{n+2 m}\right),
\end{aligned}
$$

combining with $G\left(u_{n}, u_{n+2 m-1}, u_{n+2 m}\right) \leq \frac{1+\lambda}{1-s \lambda^{2}} s \lambda^{n} G_{0}^{*}$, we obtain that

$$
G\left(u_{n}, u_{n+2 m}, u_{n+2 m+2 l+1}\right) \leq \frac{1+\lambda}{1-s \lambda^{2}} s^{2} \lambda^{n+2 m-1} G_{0}^{*}+s \lambda^{n+2 m+2 l} G_{0}^{*}+\frac{1+\lambda}{1-s \lambda^{2}} s^{2} \lambda^{n} G_{0}^{*} .
$$

Since $\lambda<\frac{1}{s}$, we can deduce that

$$
G\left(u_{n}, u_{n+2 m}, u_{n+2 m+2 l}\right) \leq \frac{3+\lambda}{1-s \lambda^{2}} s^{2} \lambda^{n} G_{0}^{*} ;
$$


Subcase 3 If $p$ is odd say $2 m+1(m \geq 0)$, and $q$ is even say $2 l(l \geq 1)$. From Definition 7 , it follows that

$$
\begin{aligned}
& G\left(u_{n}, u_{n+2 m+1}, u_{n+2 m+2 l+1}\right) \\
= & G\left(u_{n+2 m+2 l+1}, u_{n+2 m+1}, u_{n}\right) \\
\leq & s\left[G\left(u_{n+2 m+2 l+1}, u_{n+2 m+2 l+2}, u_{n+2 m+2 l+2}\right)+G\left(u_{n+2 m+2 l+2}, u_{n+2 m+2}, u_{n+2 m+2}\right)\right. \\
& \left.+G\left(u_{n+2 m+2}, u_{n+2 m+1}, u_{n}\right)\right] \\
\leq & s G\left(u_{n+2 m+2}, u_{n+2 m+2 l+1}, u_{n+2 m+2 l+2}\right)+s G_{n+2 m+2 l+1}+s G\left(u_{n}, u_{n+2 m+1}, u_{n+2 m+2}\right) \\
\leq & s^{2}\left[G_{n+2 m+2}+G_{n+2 m+3}\right]+s^{3}\left[G_{n+2 m+4}+G_{n+2 m+5}\right]+\ldots+s^{l}\left[G_{n+2 m+2 l-2}+G_{n+2 m+2 l-1}\right. \\
& \left.+G_{n+2 m+2 l}^{*}\right]+s G_{n+2 m+2 l+1}+s G\left(u_{n}, u_{n+2 m+1}, x_{n+2 m+2}\right) \\
\leq & s^{2}\left[\lambda^{n+2 m+2} G_{0}^{*}+\lambda^{n+2 m+3} G_{0}^{*}\right]+s^{3}\left[\lambda^{n+2 m+4} G_{0}^{*}+\lambda^{n+2 m+5} G_{0}^{*}\right]+\ldots+s^{l}\left[\lambda^{n+2 m+2 l-2} G_{0}^{*}\right. \\
& \left.+\lambda^{n+2 m+2 l-1} G_{0}^{*}+\lambda^{n+2 m+2 l} G_{0}^{*}\right]+s G_{n+2 m+2 l+1}+s G\left(u_{n}, u_{n+2 m+1}, u_{n+2 m+2}\right) \\
\leq & s^{2} \lambda^{n+2 m+2}\left[1+s \lambda^{2}+s^{2} \lambda^{4}+\ldots\right] G_{0}^{*}+s^{2} \lambda^{n+2 m+3}\left[1+s \lambda^{2}+s^{2} \lambda^{4}+\ldots\right] G_{0}^{*} \\
& +s G_{n+2 m+2 l+1}+s G\left(u_{n}, u_{n+2 m+1}, u_{n+2 m+2}\right) \\
= & 1+\lambda \\
& 1-s \lambda^{2} s^{2} \lambda^{n+2 m+2} G_{0}^{*}+s G_{n+2 m+2 l+1}+s G\left(u_{n}, u_{n+2 m+1}, u_{n+2 m+2}\right)
\end{aligned}
$$

and

$$
\begin{aligned}
& G\left(u_{n}, u_{n+2 m+1}, u_{n+2 m+2}\right) \\
\leq & s\left[G\left(u_{n}, u_{n+1}, u_{n+1}\right)+G\left(u_{n+1}, u_{n+2}, u_{n+2}\right)+G\left(u_{n+2}, u_{n+2 m+1}, u_{n+2 m+2}\right)\right] \\
\leq & s\left[G_{n}+G_{n+1}\right]+s^{2}\left[G_{n+2}+G_{n+3}\right]+\ldots+s^{m}\left[G_{n+2 m-2}+G_{n+2 m-1}+G_{n+2 m}^{*}\right] \\
\leq & s\left[\lambda^{n} G_{0}^{*}+\lambda^{n+1} G_{0}^{*}\right]+s^{2}\left[\lambda^{n+2} G_{0}^{*}+\lambda^{n+3} G_{0}^{*}\right]+\ldots+s^{m}\left[\lambda^{n+2 m-2} G_{0}^{*}+\lambda^{n+2 m-1} G_{0}^{*}\right. \\
& \left.+\lambda^{n+2 m} G_{0}^{*}\right] \\
\leq & s \lambda^{n}\left[1+s \lambda^{2}+s^{2} \lambda^{4}+\ldots\right] G_{0}^{*}+s \lambda^{n+1}\left[1+s \lambda^{2}+s^{2} \lambda^{4}+\ldots\right] G_{0}^{*} \\
\leq & \frac{1+\lambda}{1-s \lambda^{2}} s \lambda^{n} G_{0}^{*} .
\end{aligned}
$$

Therefore, we have

$G\left(u_{n}, u_{n+2 m+1}, u_{n+2 m+2 l+1}\right) \leq \frac{1+\lambda}{1-s \lambda^{2}} s^{2} \lambda^{n+2 m} G_{0}^{*}+s \lambda^{n+2 m+2 l} G_{0}^{*}+\frac{1+\lambda}{1-s \lambda^{2}} s^{2} \lambda^{n} G_{0}^{*}$.

Since $\lambda<\frac{1}{s}$, we can deduce that

$$
G\left(u_{n}, u_{n+2 m}, u_{n+2 m+2 l}\right) \leq \frac{3+\lambda}{1-s \lambda^{2}} s^{2} \lambda^{n} G_{0}^{*}
$$


Subcase 4 If $p$ is odd say $2 m+1(m \geq 0)$, and $q$ is odd say $2 l+1(l \geq 0)$. From Definition 7 , it follows that

$$
\begin{aligned}
& G\left(u_{n}, u_{n+2 m+1}, u_{n+2 m+2 l+2}\right) \\
= & G\left(u_{n+2 m+2 l+2}, u_{n+2 m+1}, u_{n}\right) \\
\leq & s\left[G\left(u_{n+2 m+2 l+2}, u_{n+2 m+2 l+1}, u_{n+2 m+2 l+1}\right)+G\left(u_{n+2 m+2 l+1}, u_{n+2 m+2}, u_{n+2 m+2}\right)\right. \\
& \left.+G\left(u_{n+2 m+2}, u_{n+2 m+1}, u_{n}\right)\right] \\
\leq & s\left[G\left(u_{n+2 m+2}, u_{n+2 m+2 l+1}, u_{n+2 m+2 l+2}\right)+s G_{n+2 m+2 l+1}^{\prime}+s G\left(u_{n}, u_{n+2 m+1}, u_{n+2 m+2}\right)\right] \\
\leq & s^{2}\left[G_{n+2 m+2}+G_{n+2 m+3}\right]+s^{3}\left[G_{n+2 m+4}+G_{n+2 m+5}\right]+\ldots+s^{l}\left[G_{n+2 m+2 l-2}+G_{n+2 m+2 l-1}\right. \\
& \left.+G_{n+2 m+2 l}\right]+s G_{n+2 m+2 l+1}^{\prime}+s G\left(u_{n}, u_{n+2 m+1}, u_{n+2 m+2}\right) \\
\leq & s^{2}\left[\lambda^{n+2 m+2} G_{0}^{*}+\lambda^{n+2 m+3} G_{0}^{*}\right]+s^{3}\left[\lambda^{n+2 m+4} G_{0}^{*}+\lambda^{n+2 m+5} G_{0}^{*}\right]+\ldots+s^{l}\left[\lambda^{n+2 m+2 l-2} G_{0}^{*}\right. \\
& \left.+\lambda^{n+2 m+2 l-1} G_{0}^{*}+\lambda^{n+2 m+2 l} G_{0}^{*}\right]+s \lambda^{n+2 m+2 l+1} G_{0}^{*}+s G_{n}\left(u_{n}, u_{n+2 m+1}, u_{n+2 m+2}\right) \\
\leq & s^{2} \lambda^{n+2 m+2}\left[1+s \lambda^{2}+s^{2} \lambda^{4}+\ldots\right] G_{0}^{*}+s^{2} \lambda^{n+2 m+3}\left[1+s \lambda^{2}+s^{2} \lambda^{4}+\ldots\right] G_{0}^{*} \\
& +s \lambda^{n+2 m+2 l+1} G_{0}^{*}+s G\left(u_{n}, u_{n+2 m+1}, u_{n+2 m+2}\right) \\
\leq & \frac{1+\lambda}{1-} \lambda^{2} s^{2} \lambda^{n+2 m+2} G_{0}^{*}+s \lambda^{n+2 m+2 l+1} G_{0}^{*}+s G\left(u_{n}, u_{n+2 m+1}, u_{n+2 m+2}\right),
\end{aligned}
$$

combining with $G\left(u_{n}, u_{n+2 m+1}, u_{n+2 m+2}\right) \leq \frac{1+\lambda}{1-s \lambda^{2}} s \lambda^{n} G_{0}^{*}$, we obtain that

$$
G\left(u_{n}, u_{n+2 m}, u_{n+2 m+2 l+1}\right) \leq \frac{1+\lambda}{1-s \lambda^{2}} s^{2} \lambda^{n+2 m} G_{0}^{*}+s \lambda^{n+2 m+2 l+1} G_{0}^{*}+\frac{1+\lambda}{1-s \lambda^{2}} s^{2} \lambda^{n} G_{0}^{*} .
$$

Since $\lambda<\frac{1}{s}$, we can deduce that

$$
G\left(u_{n}, u_{n+2 m}, u_{n+2 m+2 l}\right) \leq \frac{3+\lambda}{1-s \lambda^{2}} s^{2} \lambda^{n} G_{0}^{*}
$$

In view of the all above subcases, we obtain that

$$
G\left(u_{n}, u_{n+2 m}, u_{n+2 m+2 l}\right) \leq \frac{3+\lambda}{1-s \lambda^{2}} s^{2} \lambda^{n} G_{0}^{*},
$$

for all $n \in \mathbb{N}$. We reach Case1, thus $\left\{u_{n}\right\}$ is a $G-$ Cauchy sequence in $E$.

Case 2 Let $\lambda \in\left[\frac{1}{s}, 1\right)(s>1)$. Since $\lim _{n \rightarrow \infty} \lambda^{n}=0$, there exists $N_{0} \in N$ such that $\lambda^{N_{0}}<\frac{1}{s}$. Thus, by Case 1 , we claim that

$$
\left\{\left(T^{N_{0}}\right)^{n} u_{0}\right\}_{n=0}^{\infty}:=\left\{u_{N_{0}}, u_{N_{0}+1}, \ldots, u_{N_{0}+n}, \ldots\right\}
$$

is $G$-Cauchy sequence. Since

$$
\left\{u_{n}\right\}_{n=0}^{\infty}=\left\{u_{1}, u_{2}, \ldots, u_{N_{0}}\right\} \cup\left\{u_{N_{0}+1}, u_{N_{0}+2}, \ldots, u_{N_{0}+n}, \ldots\right\},
$$

we conclude that the sequence $\left\{u_{n}\right\}$ is a $G$-Cauchy sequence.

Case 3 Let $s=1$. Similar to the process of Case 1, the claim holds.

Next, we will give some fixed point theorems. Firstly, we now state and prove the Banach contraction principle in $R G_{b} M S$.

Theorem 1. Let $(E, G)$ be a complete $R G_{b} M S$ with coefficient $s \geq 1$ and the mapping $T: E \rightarrow E$ satisfies the contractive condition:

$$
G(T u, T v, T o) \leq \lambda G(u, v, o), \text { for all } u, v, o \in E,
$$


where $\lambda \in[0,1)$ is a constant. Then $T$ has a unique fixed point in $E$.

Proof. Let $u_{0} \in E$ and define a sequence $\left\{u_{n}\right\}$ in $E$ by $u_{n}=T u_{n-1}$ for all $n \in \mathbb{N}$. Using the inequality (3), we have

$$
G\left(u_{n}, u_{n+1}, u_{n+2}\right)=G\left(T u_{n-1}, T u_{n}, T u_{n+1}\right) \leq \lambda G\left(u_{n-1}, u_{n}, u_{n+1}\right) .
$$

If $u_{n}=u_{n+1}$ then $u_{n}$ is fixed point of $T$ and the proof is completed. So, suppose that $u_{n} \neq u_{n+1}$ for all $n \in \mathbb{N}$. Also, we can assume that $u_{n} \neq u_{n+p}$ for all $p \geq 2$. Indeed, if $u_{n}=u_{n+p}$, then using the inequality (3), we get

$$
\begin{aligned}
G\left(u_{n}, u_{n+1}, u_{n+1}\right) & =G\left(u_{n}, T u_{n}, T u_{n}\right) \\
& =G\left(T u_{n+p-1}, T u_{n+p}, T u_{n+p}\right) \\
& \leq \lambda^{p-1} G\left(u_{n}, u_{n+1}, u_{n+1}\right),
\end{aligned}
$$

a contradiction. Therefore, in what follows, we assume that $u_{n} \neq u_{m}$ for all $n \neq m$. Thus, similarly to the proof of Lemma 3, we conclude that $\left\{u_{n}\right\}$ is a $G$-Cauchy sequence in $E$. Since $(E, G)$ is a complete $R G_{b} M S$, hence, for any $\varepsilon>0$, there exists $n_{0} \in \mathbb{N}$ and $u^{*} \in E$ such that $G\left(u_{n}, u_{m}, u^{*}\right)<\frac{\varepsilon}{3 s}$ for all $n, m \geq n_{0}$. Moreover, it follows from Proposition 1 that $G\left(u^{*}, u_{n}, u_{n}\right)<\frac{\varepsilon}{3 s}, G\left(u_{n}, u_{n-1}, u_{n-1}\right)<\frac{\varepsilon}{3 s}$ and $G\left(u_{n}, u^{*}, u^{*}\right)<\frac{\varepsilon}{3 s}$, for all $n \geq n_{0}$. Then, we have

$$
\begin{aligned}
G\left(u^{*}, T u^{*}, T u^{*}\right) & \leq s\left[G\left(u^{*}, u_{n}, u_{n}\right)+G\left(u_{n}, u_{n+1}, u_{n+1}\right)+G\left(u_{n+1}, T u^{*}, T u^{*}\right)\right] \\
& \leq s\left[G\left(u^{*}, u_{n}, u_{n}\right)+G\left(u_{n}, u_{n+1}, u_{n+1}\right)+\lambda G\left(u_{n}, u^{*}, u^{*}\right)\right] \\
& <s\left[\left(\frac{\varepsilon}{3 s}\right)+\left(\frac{\varepsilon}{3 s}\right)+\lambda\left(\frac{\varepsilon}{3 s}\right)\right] \\
& <\varepsilon
\end{aligned}
$$

which implies $G\left(u^{*}, T u^{*}, T u^{*}\right)=0$. Therefore, $T u^{*}=u^{*}$ and $u^{*}$ is a fixed point of $T$. Now we show that the fixed point $u^{*}$ is unique. If there is another fixed point $v^{*} \neq u^{*}$, by the given condition (3), we have

$$
G\left(u^{*}, v^{*}, v^{*}\right)=G\left(T u^{*}, T v^{*}, T v^{*}\right) \leq \lambda G\left(u^{*}, v^{*}, v^{*}\right),
$$

which is a contradiction. Thus, $u^{*}$ is a unique fixed point of $T$.

The following theorem is a Ćirić type fixed point result in a $R G_{b} M S$.

Theorem 2. Let $(E, G)$ be a complete $R G_{b} M S$ with coefficient $s \geq 1$ and the mapping $T: E \rightarrow E$ satisfies the contractive condition:

$$
G(T u, T v, T o) \leq \lambda M_{u, v, o}
$$

where

$$
\begin{array}{r}
M_{u, v, o}=\max \{G(u, v, o), G(u, T u, T u), G(v, T v, T v), G(o, T o, T o), G(u, T v, T v), \\
G(u, T o, T o), G(v, T u, T u), G(v, T o, T o), G(o, T u, T u), G(o, T v, T v)\}
\end{array}
$$

for any $u, v, o \in E$, where $0 \leq \lambda<\frac{1}{3 s^{2}}$. Then $T$ has a unique fixed point in $E$.

Proof. Let $u_{0} \in E$ and define a sequence $\left\{u_{n}\right\}$ in $E$ by $u_{n}=T u_{n-1}$ for all $n \in \mathbb{N}$. If $u_{n}=u_{n+1}$, then $u_{n}$ is a fixed point of $T$ and the proof is completed. So, suppose that $u_{n} \neq u_{n+1}$ for all $n \in \mathbb{N}$. It follows from the inequality (4) that

$$
G\left(u_{n}, u_{n+1}, u_{n+2}\right)=G\left(T u_{n-1}, T u_{n}, T u_{n+1}\right) \leq \lambda M_{u_{n-1}, u_{n}, u_{n+1}} .
$$


Noting that

$$
\begin{aligned}
M_{u_{n-1}, u_{n}, u_{n+1}=\max \{} & G\left(u_{n-1}, u_{n}, u_{n+1}\right), G\left(u_{n-1}, T u_{n-1}, T u_{n-1}\right), G\left(u_{n}, T u_{n}, T u_{n}\right), \\
& G\left(u_{n+1}, T u_{n+1}, T u_{n+1}\right), G\left(u_{n-1}, T u_{n}, T u_{n}\right), G\left(u_{n-1}, T u_{n+1}, T u_{n+1}\right), \\
& G\left(u_{n}, T u_{n+1}, T u_{n+1}\right), G\left(u_{n}, T u_{n-1}, T u_{n-1}\right), G\left(u_{n+1}, T u_{n-1}, T u_{n-1}\right), \\
& \left.G\left(u_{n+1}, T u_{n}, T u_{n}\right)\right\} \\
=\max \{ & G\left(u_{n-1}, u_{n}, u_{n+1}\right), G\left(u_{n-1}, u_{n}, u_{n}\right), G\left(u_{n}, u_{n+1}, u_{n+1}\right), \\
& G\left(u_{n+1}, u_{n+2}, u_{n+2}\right), G\left(u_{n-1}, u_{n+1}, u_{n+1}\right), G\left(u_{n-1}, u_{n+2}, u_{n+2}\right), \\
& \left.G\left(u_{n}, u_{n+2}, u_{n+2}\right), G\left(u_{n+1}, u_{n}, u_{n}\right)\right\} .
\end{aligned}
$$

Using (RGb3) and (RGb5) in the above inequality, we obtain

$$
\begin{aligned}
M_{u_{n-1}, u_{n}, u_{n+1}=} \max \left\{G\left(u_{n-1}, u_{n}, u_{n+1}\right), G\left(u_{n+1}, u_{n+2}, u_{n+2}\right), G\left(u_{n-1}, u_{n+2}, u_{n+2}\right),\right. & \\
& \left.G\left(u_{n}, u_{n+2}, u_{n+2}\right)\right\} \\
\leq & \max \left\{G\left(u_{n-1}, u_{n}, u_{n+1}\right), G\left(u_{n-1}, u_{n}, u_{n}\right), G\left(u_{n+1}, u_{n+2}, u_{n+2}\right),\right. \\
& s\left[G\left(u_{n-1}, u_{n}, u_{n}\right)+G\left(u_{n}, u_{n+1}, u_{n+1}\right)+G\left(u_{n+1}, u_{n+2}, u_{n+2}\right)\right], \\
& \left.G\left(u_{n}, u_{n+2}, u_{n+2}\right)\right\} \\
\leq & \max \left\{G\left(u_{n-1}, u_{n}, u_{n+1}\right), G\left(u_{n-1}, u_{n}, u_{n}\right), G\left(u_{n+1}, u_{n+2}, u_{n+2}\right),\right. \\
& \left.\quad 2 s G\left(u_{n-1}, u_{n}, u_{n+1}\right)+s G\left(u_{n}, u_{n+1}, u_{n+2}\right), G\left(u_{n}, u_{n+2}, u_{n+2}\right)\right\} \\
= & 2 s G\left(u_{n-1}, u_{n}, u_{n+1}\right)+s G\left(u_{n}, u_{n+1}, u_{n+2}\right) .
\end{aligned}
$$

Therefore, we get

$$
G\left(u_{n}, u_{n+1}, u_{n+2}\right) \leq 2 s \lambda G\left(u_{n-1}, u_{n}, u_{n+1}\right)+s \lambda G\left(u_{n}, u_{n+1}, u_{n+2}\right) .
$$

As $\lambda \in\left[0, \frac{1}{3 s^{2}}\right)$, it follows from the above inequality that

$$
G\left(u_{n}, u_{n+1}, u_{n+2}\right) \leq \frac{2 s \lambda}{1-s \lambda} G\left(u_{n-1}, u_{n}, u_{n+1}\right) .
$$

Let $k=\frac{2 s \lambda}{1-s \lambda}$, it is clear that $k<1$. We also can assume that $u_{n} \neq u_{n+p}$ for all $n \in \mathbb{N}$ and $p \geq 2$. Indeed, if $u_{n}=u_{n+p}$, using the inequality (5), we have

$$
\begin{aligned}
G\left(u_{n}, u_{n+1}, u_{n+2}\right) & =G\left(u_{n+p}, u_{n+1}, u_{n+2}\right) \\
& \leq k G\left(u_{n+p-1}, u_{n}, u_{n+1}\right) \\
& =k G\left(u_{n+p-1}, u_{n+p}, u_{n+1}\right) \\
& \leq k^{2} G\left(u_{n+p-2}, u_{n+p-1}, u_{n}\right) \\
& \leq \ldots \leq k^{p} G\left(u_{n}, u_{n+1}, u_{n+2}\right),
\end{aligned}
$$

a contradiction. Thus, similarly to the proof of Lemma 3, we conclude that $\left\{u_{n}\right\}$ is a $G$-Cauchy sequence in $E$. Since $(E, d)$ is a complete $R G_{b} M S$, hence, for any $\varepsilon>0$, there exists $n_{0} \in \mathbb{N}$ and $u^{*} \in E$ such that $G\left(u_{n}, u_{m}, u^{*}\right)<\frac{1-s^{2} \lambda}{4 s} \varepsilon$ for all $n, m \geq n_{0}$. Moreover, it follows from Proposition 1 that $G\left(u^{*}, u_{n}, u_{n}\right)<\frac{1-s^{2} \lambda}{4 s} \varepsilon, G\left(u_{n}, u^{*}, u^{*}\right)<\frac{1-s^{2} \lambda}{4 s} \varepsilon$, $G\left(u_{n}, u_{n+1}, u_{n+1}\right)<\frac{1-s^{2} \lambda}{4 s} \varepsilon$ and $G\left(u_{n+1}, u_{n}, u_{n}\right)<\frac{1-s^{2} \lambda}{4 s} \varepsilon$ for all $n \geq n_{0}$. For any $n \in \mathbb{N}$, we have

$$
\begin{aligned}
G\left(u^{*}, T u^{*}, T u^{*}\right) & \leq s\left[G\left(u^{*}, u_{n+1}, u_{n+1}\right)+G\left(u_{n+1}, u_{n+2}, u_{n+2}\right)+G\left(u_{n+2}, T u^{*}, T u^{*}\right)\right] \\
& \leq s G\left(u^{*}, u_{n+1}, u_{n+1}\right)+s G\left(u_{n+1}, u_{n+2}, u_{n+2}\right)+s \lambda M_{u_{n+1}, u^{*}, u^{*}}
\end{aligned}
$$


and

$$
\begin{gathered}
M_{u_{n+1}, u^{*}, u^{*}}=\max \left\{G\left(u_{n+1}, u^{*}, u^{*}\right), G\left(u_{n+1}, T u_{n+1}, T u_{n+1}\right), G\left(u^{*}, T u^{*}, T u^{*}\right),\right. \\
\left.G\left(u_{n+1}, T u^{*}, T u^{*}\right), G\left(u^{*}, T u_{n+1}, T u_{n+1}\right)\right\} \\
=\max \left\{G\left(u_{n+1}, u^{*}, u^{*}\right), G\left(u_{n+1}, T u_{n+1}, T u_{n+1}\right), G\left(u^{*}, T u^{*}, T u^{*}\right),\right. \\
\left.G\left(u_{n+1}, T u^{*}, T u^{*}\right), G\left(u^{*}, u_{n+2}, u_{n+2}\right)\right\} .
\end{gathered}
$$

If $M_{u_{n+1}, u^{*}, u^{*}}=G\left(u^{*}, T u^{*}, T u^{*}\right)$, then we have

$$
G\left(u^{*}, T u^{*}, T u^{*}\right) \leq s G\left(u^{*}, u_{n+1}, u_{n+1}\right)+s G\left(u_{n+1}, u_{n+2}, u_{n+2}\right)+s \lambda G\left(u^{*}, T u^{*}, T u^{*}\right),
$$

since $s \lambda<1$, the above inequality yields

$$
\begin{aligned}
& G\left(u^{*}, T u^{*}, T u^{*}\right) \leq \frac{s}{1-s \lambda}\left[G\left(u^{*}, u_{n+1}, u_{n+1}\right)+G\left(u_{n+1}, u_{n+2}, u_{n+2}\right)\right]<\frac{\varepsilon}{4}+\frac{\varepsilon}{4}<\varepsilon ; \\
& \text { If } M_{u_{n+1}, u^{*}, u^{*}=} G\left(u_{n+1}, T u^{*}, T u^{*}\right), \text { we have } \\
& \begin{aligned}
G\left(u^{*}, T u^{*}, T u^{*}\right) \leq & s G\left(u^{*}, u_{n+1}, u_{n+1}\right)+s G\left(u_{n+1}, u_{n+2}, u_{n+2}\right)+s \lambda G\left(u_{n+1}, T u^{*}, T u^{*}\right) \\
\leq & s G\left(u^{*}, u_{n+1}, u_{n+1}\right)+s G\left(u_{n+1}, u_{n+2}, u_{n+2}\right)+s^{2} \lambda\left[G\left(u_{n+1}, u_{n}, u_{n}\right)\right. \\
& \left.+G\left(u_{n}, u^{*}, u^{*}\right)+G\left(u^{*}, T u^{*}, T u^{*}\right)\right],
\end{aligned}
\end{aligned}
$$

since $s^{2} \lambda<1$, the above inequality yields

$$
\begin{aligned}
G\left(u^{*}, T u^{*}, T u^{*}\right) \leq & \frac{1}{1-s^{2} \lambda}\left[s G\left(u^{*}, u_{n+1}, u_{n+1}\right)+s G\left(u_{n+1}, u_{n+2}, u_{n+2}\right)\right. \\
& \left.+s^{2} \lambda G\left(u_{n+1}, u_{n}, u_{n}\right)+s^{2} \lambda G\left(u_{n}, u^{*}, u^{*}\right)\right] \\
< & \frac{\varepsilon}{4}+\frac{\varepsilon}{4}+\frac{\varepsilon}{4}+\frac{\varepsilon}{4}=\varepsilon ;
\end{aligned}
$$

If $M_{u_{n+1}, u^{*}, u^{*}}=\max \left\{G\left(u_{n+1}, u^{*}, u^{*}\right), G\left(u_{n+1}, T u_{n+1}, T u_{n+1}\right), G\left(u^{*}, u_{n+2}, u_{n+2}\right)\right\}$, which implies that $M_{u_{n+1}, u^{*}, u^{*}}<\frac{1-s^{2} \lambda}{4 s} \varepsilon$. Hence, we have

$$
G\left(u^{*}, T u^{*}, T u^{*}\right)<\frac{\varepsilon}{4}+\frac{\varepsilon}{4}+\frac{\varepsilon}{4}<\varepsilon .
$$

Consequently, we deduce that $G\left(u^{*}, T u^{*}, T u^{*}\right)=0$, hence, $T u^{*}=u^{*}$ and $u^{*}$ is a fixed point of $T$. To prove the uniqueness of the fixed point, suppose that $v^{*}$ is another fixed point of $T$. We have

$$
\begin{aligned}
G\left(u^{*}, v^{*}, v^{*}\right) & =G\left(T u^{*}, T v^{*}, T v^{*}\right) \\
& \leq \lambda \max \left\{G\left(u^{*}, v^{*}, v^{*}\right), G\left(u^{*}, T v^{*}, T v^{*}\right), G\left(v^{*}, T u^{*}, T u^{*}\right)\right\} \\
& \leq \lambda G\left(u^{*}, v^{*}, v^{*}\right),
\end{aligned}
$$

a contradiction. Hence, $u^{*}=v^{*}$ which completes the proof.

As a similar consequence of Theorem 2, we obtain the following result.

Corollary 1. Let $(E, G)$ be a complete $R G_{b} M S$ with coefficient $s \geq 1$ and the mapping $T: E \rightarrow E$ satisfies the contractive condition:

$$
G(T u, T v, T o) \leq \lambda M_{u, v, o}
$$


where

$$
\begin{array}{r}
M_{u, v, o}=\max \{G(u, v, o), G(u, u, T u), G(v, v, T v), G(o, o, T o), G(u, u, T v), \\
G(u, u, T o), G(v, v, T u), G(v, v, T o), G(o, o, T u), G(o, o, T v)\}
\end{array}
$$

for any $u, v, o \in E$, where $0 \leq \lambda<\frac{1}{3 s^{2}}$. Then $T$ has a unique fixed point in $E$.

The following theorem is a Reich type fixed point result in a $R G_{b} M S$.

Theorem 3. Let $(E, G)$ be a complete $R G_{b} M S$ with coefficient $s \geq 1$ and the mapping $T: E \rightarrow E$ satisfies the contractive condition:

$$
G(T u, T v, T o) \leq a_{1} G(u, v, o)+a_{2} G(u, T u, T u)+a_{3} G(v, T v, T v)+a_{4} G(o, T o, T o)
$$

for all $u, v, o \in E$, where $a_{1}+a_{2}+a_{3}+a_{4}<1$ and $s\left(a_{3}+a_{4}\right)<1$. Then $T$ has a unique fixed point in $E$.

Proof. Let $u_{0} \in E$ and define a sequence $\left\{u_{n}\right\}$ in $E$ by $u_{n}=T u_{n-1}$ for all $n \in \mathbb{N}$. If $u_{n}=u_{n+1}$ then $u_{n}$ is fixed point of $T$ and the proof is completed. So, suppose that $u_{n} \neq u_{n+1}$ for all $n \in \mathbb{N}$. It follows from the inequality (6) that

$$
\begin{aligned}
& G\left(u_{n}, u_{n+1}, u_{n+2}\right) \\
= & G\left(T u_{n-1}, T u_{n}, T u_{n+1}\right) \\
\leq & a_{1} G\left(u_{n-1}, u_{n}, u_{n+1}\right)+a_{2} G\left(u_{n-1}, T u_{n-1}, T u_{n-1}\right)+a_{3} G\left(u_{n}, T u_{n}, T u_{n}\right) \\
& +a_{4} G\left(u_{n+1}, T u_{n+1}, T u_{n+1}\right) \\
= & a_{1} G\left(u_{n-1}, u_{n}, u_{n+1}\right)+a_{2} G\left(u_{n-1}, u_{n}, u_{n}\right)+a_{3} G\left(u_{n}, u_{n+1}, u_{n+1}\right)+a_{4} G\left(u_{n+1}, u_{n+2}, u_{n+2}\right) \\
\leq & a_{1} G\left(u_{n-1}, u_{n}, u_{n+1}\right)+a_{2} G\left(u_{n-1}, u_{n}, u_{n+1}\right)+a_{3} G\left(u_{n}, u_{n+1}, u_{n+2}\right)+a_{4} G\left(u_{n}, u_{n+1}, u_{n+2}\right) .
\end{aligned}
$$

Hence, we get

$$
G\left(u_{n}, u_{n+1}, u_{n+2}\right) \leq \frac{a_{1}+a_{2}}{1-a_{3}-a_{4}} G\left(u_{n-1}, u_{n}, u_{n+1}\right) .
$$

Let $k=\frac{a_{1}+a_{2}}{1-a_{3}-a_{4}}$, where $0 \leq k<1$. Also, we can assume that $u_{n} \neq u_{n+p}$ for all $p \geq 2$. Indeed, if $u_{n}=u_{n+p}$, then using the inequality (6), we get

$$
\begin{aligned}
G\left(u_{n}, u_{n+1}, u_{n+1}\right) & =G\left(u_{n}, T u_{n}, T u_{n}\right) \\
& =G\left(T u_{n+p-1}, T u_{n+p}, T u_{n+p}\right) \\
& \leq k^{p-1} G\left(u_{n}, u_{n+1}, u_{n+1}\right),
\end{aligned}
$$

a contradiction. In what follows, we can assume that $u_{n} \neq u_{m}$ for all $n \neq m$. Thus, similarly to the proof of Lemma 3, we conclude that $\left\{u_{n}\right\}$ is a $G$-Cauchy sequence in $E$. Since $(E, G)$ is a complete $R G_{b} M S$, hence, for any $\varepsilon>0$, there exists $n_{0} \in \mathbb{N}$ and $u^{*} \in E$ such that $G\left(u^{*}, u_{n}, u_{m}\right)<\frac{\varepsilon}{3\left(1+a_{1}+a_{2}\right) s}$ for all $n, m \geq n_{0}$. Moreover, it follows from Proposition 1 that $G\left(u^{*}, u_{n}, u_{n}\right)<\frac{\varepsilon}{3 s}, G\left(u_{n}, u_{n+1}, u_{n+1}\right)<\frac{\varepsilon}{3\left(1+a_{2}\right) s}$ and $G\left(u_{n}, u^{*}, u^{*}\right)<\frac{\varepsilon}{3 a_{1} s}$ for all $n \geq n_{0}$. For any $n \in \mathbb{N}$, we have

$$
\begin{aligned}
G\left(u^{*}, T u^{*}, T u^{*}\right) \leq & s\left[G\left(u^{*}, u_{n}, u_{n}\right)+G\left(u_{n}, u_{n+1}, u_{n+1}\right)+G\left(u_{n+1}, T u^{*}, T u^{*}\right)\right] \\
\leq & s G\left(u^{*}, u_{n}, u_{n}\right)+s G\left(u_{n}, u_{n+1}, u_{n+1}\right)+s a_{1} G\left(u_{n}, u^{*}, u^{*}\right) \\
& +s a_{2} G\left(u_{n}, T u_{n}, T u_{n}\right)+s a_{3} G\left(u^{*}, T u^{*}, T u^{*}\right)+s a_{4} G\left(u^{*}, T u^{*}, T u^{*}\right) .
\end{aligned}
$$


Since $s\left(a_{3}+a_{4}\right)<1$, the above inequality yields

$$
\begin{aligned}
G\left(u^{*}, T u^{*}, T u^{*}\right) \leq & \frac{s}{1-s\left(a_{3}+a_{4}\right)}\left[G\left(u^{*}, u_{n}, u_{n}\right)+\left(1+a_{2}\right) G\left(u_{n}, u_{n+1}, u_{n+1}\right)\right. \\
& \left.+a_{1} G\left(u_{n}, u^{*}, u^{*}\right)\right],
\end{aligned}
$$

we obtain

$$
G\left(u^{*}, T u^{*}, T u^{*}\right)<\frac{s\left(\frac{\varepsilon}{3}+\frac{\varepsilon}{3}+\frac{\varepsilon}{3}\right)}{\left(1-s\left(a_{3}+a_{4}\right)\right)}=\frac{s \varepsilon}{\left(1-s\left(a_{3}+a_{4}\right)\right)} .
$$

We deduce that $G\left(u^{*}, T u^{*}, T u^{*}\right)=0$, that is, $T u^{*}=u^{*}$. So $u^{*}$ is a fixed point of $T$. Now we show that the fixed point $u^{*}$ is unique. If there is another fixed point $v^{*} \neq u^{*}$, by the given condition (6), we have

$$
\begin{aligned}
G\left(u^{*}, v^{*}, v^{*}\right)= & G\left(T u^{*}, T v^{*}, T v^{*}\right) \\
\leq & a_{1} G\left(u^{*}, v^{*}, v^{*}\right)+a_{2} G\left(u^{*}, T u^{*}, T u^{*}\right)+a_{3} G\left(v^{*}, T v^{*}, T v^{*}\right) \\
& +a_{4} G\left(v^{*}, T v^{*}, T v^{*}\right) \\
= & a_{1} G\left(u^{*}, v^{*}, v^{*}\right),
\end{aligned}
$$

which is a contradiction. Hence, $u^{*}=v^{*}$ which completes the proof.

As a similar consequence of Theorem 3, we obtain the following result.

Corollary 2. Let $(E, G)$ be a complete $R G_{b} M S$ with coefficient $s \geq 1$ and the mapping $T: E \rightarrow E$ satisfies the contractive condition:

$$
G(T u, T v, T o) \leq a_{1} G(u, v, o)+a_{2} G(u, u, T u)+a_{3} G(v, v, T v)+a_{4} G(o, o, T o)
$$

for all $u, v, o \in E$, where $a_{1}+a_{2}+a_{3}+a_{4}<1$ and $s\left(a_{3}+a_{4}\right)<1$. Then $T$ has a unique fixed point in $E$.

\section{Convex Rectangular $G_{b}$-Metric Spaces}

In this section, we will give the notion of convex rectangular $G_{b}$-metric space and prove several fixed point theorems for enriched type contractions in this space.

Definition 11 ([19]). Let $(E, d)$ be a metric space and $I=[0,1]$. A continuous function $w$ : $E \times E \times[0,1] \rightarrow E$ is said to be a convex structure on $E$ if for each $u, v, o \in E$ and $\alpha \in I$,

$$
d(o, w(u, v ; \alpha)) \leq \alpha d(o, u)+(1-\alpha) d(o, v)
$$

holds. A metric space $(E, d)$ with a convex structure $w$ is called a convex metric space.

Next, we introduce the notion of convex rectangular $G_{b}$-metric space.

Definition 12. Let $(E, G)$ be a complete $R G_{b} M S$ with coefficient $s \geq 1$. A mapping $w: E \times E \times$ $[0,1] \rightarrow E$ is said to be a convex structure on $E$ if for each $u, v, z, h \in E$ and $\alpha \in I$,

$$
G(u, v, w(z, h ; \alpha)) \leq \alpha G(u, v, z)+(1-\alpha) G(u, v, h)
$$

holds. Then the triplet $(E, G, w)$ is called a convex rectangular $G_{b}$-metric space $\left(C R G_{b} M S\right)$ with coefficient $s \geq 1$.

Let us present now a specific example of $C R G_{b} M S$.

Example 8. Let $E=\mathbb{R}$ and for any $u, v, o \in E$, let us defined $G$ by

$$
G(u, v, o)=(|u-v|+|v-o|+|u-o|)^{2},
$$


as well as the mapping $w: E \times E \times[0,1] \rightarrow E$ by the formula

$$
w(u, v ; \alpha)=\alpha u+(1-\alpha) v .
$$

Then $(E, G, w)$ is a $C R G_{b} M S$ with $s=3$. Indeed, Example 5 shows us that $(E, G)$ is a $R G_{b} M S$ with $s=3$. We only need to verify that $G$ satisfies inequality (7). For any $u, v, z, h \in E$, we get

$$
\begin{aligned}
& G_{b}(u, v, w(z, h ; \alpha)) \\
= & {[|u-v|+|v-\alpha z-(1-\alpha) h|+|u-\alpha z-(1-\alpha) h|]^{2} } \\
\leq & {[\alpha|u-v|+(1-\alpha)|u-v|+\alpha|v-z|+(1-\alpha)|v-h|+\alpha|u-z|+(1-\alpha)|u-h|]^{2} } \\
= & {[\alpha(|u-v|+|v-z|+|u-z|)+(1-\alpha)(|u-v|+|v-h|+|u-h|)]^{2} } \\
= & \alpha^{2} G(u, v, z)+2 \alpha(1-\alpha)(|u-v|+|v-z|+|u-z|)(|u-v|+|v-h|+|u-h|) \\
& +(1-\alpha)^{2} G(u, v, h) \\
\leq & \alpha^{2} G(u, v, z)+2 \alpha(1-\alpha) \frac{G(u, v, z)+G(u, v, h)}{2}+(1-\alpha)^{2} G(u, v, h) \\
= & \alpha G(u, v, z)+(1-\alpha) G(u, v, h)
\end{aligned}
$$

Therefore, $(E, G, w)$ is a $C R G_{b} M S$ with $s=3$.

In what follows, we denote the set of all fixed points of $T$ by $F(T)$, that is,

$$
F(T)=\{u \in E: T u=u\}
$$

The next Lemma is a partial extension of a result given in [27] from Banach spaces to rectangular $G_{b}$-metric spaces.

Lemma 4. Let $(E, G, w)$ be a $C R G_{b} M S$ and $T: E \rightarrow E$ be a mapping. Define the mapping $T_{\alpha}$ by

$$
T_{\alpha} u=w(u, T u ; \alpha)
$$

for all $u \in E$. Then, for any $\alpha \in[0,1)$,

$$
F(T)=F\left(T_{\alpha}\right)
$$

Proof. If $\alpha=0$, then

$$
G\left(T u, T u, T_{\alpha} u\right)=G(T u, T u, w(u, T u ; 0)) \leq G(T u, T u, T u)=0
$$

which implies that $T u=T_{\alpha} u$. If $\alpha \in(0,1)$, let $u^{*} \in F(T)$, that is, $u^{*}=T u^{*}$ and we get

$$
G\left(u^{*}, u^{*}, T_{\alpha} u^{*}\right)=G\left(u^{*}, u^{*}, w\left(u^{*}, T u^{*} ; \alpha\right)\right) \leq(1-\alpha) G\left(u^{*}, u^{*}, T u^{*}\right)=0
$$

which means $u^{*} \in F\left(T_{\alpha} u^{*}\right)$. Conversely, let $u^{*} \in F\left(T_{\alpha}\right)$, that is, $u^{*}=T_{\alpha} u^{*}$ and we obtain

$$
G\left(T u^{*}, T u^{*}, u^{*}\right)=G\left(T u^{*}, T u^{*}, w\left(u^{*}, T u^{*} ; \alpha\right)\right) \leq \alpha G\left(T u^{*}, T u^{*}, u^{*}\right)=0
$$

which means $u^{*} \in F\left(T u^{*}\right)$.

Let $\Theta$ be the set of non-decreasing function function $\varphi:[0, \infty) \longrightarrow[0, \infty)$ such that $\lim _{n \rightarrow \infty} \varphi^{n}(t)=0$ for all $t \geq 0$. If $\varphi \in \Theta$, it follows from Berinde [28] that

(1) $\varphi(0)=0$;

(2) $\varphi(t)<t$ for all $t>0$.

Now we give the notion of enriched $\varphi$-contraction in a $C R G_{b} M S$. 
Definition 13. Let $(E, G, w)$ be a $C R G_{b} M S$ with $s \geq 1$. A mapping $T: E \rightarrow E$ is said to be an enriched $\varphi$-contraction if there exists $\varphi \in \Theta$ and $\alpha \in[0,1)$ such that

$$
G(w(u, T u ; \alpha), w(v, T v ; \alpha), w(o, T o ; \alpha)) \leq \frac{1}{s^{2}} \varphi(G(u, v, o))
$$

for all $u, v, o \in E$.

Theorem 4. Let $(E, G, w)$ be a $C R G_{b} M S$ with $s \geq 1$ and let $T: E \rightarrow E$ be an enriched $\varphi-$ contraction. Then

(1) $F(T)=\left\{u^{*}\right\}$, for some $u^{*} \in E$;

(2) the sequence $\left\{u_{n}\right\}$ obtained from the iterative process

$$
u_{n+1}=w\left(u_{n}, T u_{n} ; \alpha_{n}\right)
$$

converges to $u^{*}$, for any $u_{0} \in E$.

Proof. Let us define the mapping $T_{\alpha}: E \longrightarrow E$ by $T_{\alpha} u:=w(u, T u ; \alpha)$. Then the inequality (8) can be rewritten as

$$
G\left(T_{\alpha} u, T_{\alpha} v, T_{\alpha} o\right) \leq \frac{1}{s^{2}} \varphi(G(u, v, o)) .
$$

Let $u_{0} \in E$ and $u_{n}=T_{\alpha_{n-1}} u_{n-1}$ where $T_{\alpha_{n}} u_{n}=w\left(u_{n}, T u_{n} ; \alpha_{n}\right)$ for all $n \in \mathbb{N}$. If $u_{n}=u_{n+1}$, then $u_{n}$ is a fixed point of $T_{\alpha}$. So, suppose that $u_{n} \neq u_{n+1}$ for all $n \in \mathbb{N}$. Then, it follows from inequality (9) that

$$
\begin{aligned}
G\left(u_{n}, u_{n+1}, u_{n+2}\right) & \leq \frac{1}{s^{2}} \varphi\left(G\left(u_{n-1}, u_{n}, u_{n+1}\right)\right) \\
& \leq \varphi\left(G\left(u_{n-1}, u_{n}, u_{n+1}\right)\right) \\
& \leq \ldots \leq \varphi^{n}\left(G\left(u_{0}, u_{1}, u_{2}\right)\right)
\end{aligned}
$$

then we have

$$
\lim _{n \rightarrow \infty} G\left(u_{n}, u_{n+1}, u_{n+2}\right)=0 .
$$

Also, we can assume $u_{n} \neq u_{n+p}$ for any $p>1$. Indeed, if $u_{n}=u_{n+p}$, then using the inequality (9), we have

$$
\begin{aligned}
G\left(u_{n}, u_{n}, u_{n+1}\right) & =G\left(u_{n+p}, u_{n+p}, u_{n+1}\right) \\
& \leq \frac{1}{s^{2}} \varphi\left(G\left(u_{n+p-1}, u_{n+p-1}, u_{n}\right)\right) \\
& \leq \varphi\left(G\left(u_{n+p-1}, u_{n+p-1}, u_{n+p}\right)\right) \\
& \leq \varphi^{2}\left(G\left(u_{n+p-2}, u_{n+p-2}, u_{n+p-1}\right)\right) \\
& \leq \ldots \\
& \leq \varphi^{p}\left(G\left(u_{n}, u_{n}, u_{n+1}\right)\right) \\
& <G\left(u_{n}, u_{n}, u_{n+1}\right),
\end{aligned}
$$

a contradiction. Notice that

$$
\begin{aligned}
G\left(u_{n}, u_{n+2}, u_{n+2}\right) & =G\left(T_{\alpha_{n-1}} u_{n-1}, T_{\alpha_{n+1}} u_{n+1}, T_{\alpha_{n+1}} u_{n+1}\right) \\
& \leq \frac{1}{s^{2}} \varphi\left(G\left(u_{n-1}, u_{n+1}, u_{n+1}\right)\right) \\
& \leq \varphi\left(G\left(u_{n-1}, u_{n+1}, u_{n+1}\right)\right) \\
& \leq \ldots \leq \varphi^{n}\left(G\left(u_{0}, u_{2}, u_{2}\right)\right) .
\end{aligned}
$$


It follows that

$$
G\left(u_{n}, u_{n+2}, u_{n+2}\right) \leq \varphi^{n}\left(G\left(u_{0}, u_{2}, u_{2}\right)\right) .
$$

Similary, we can conclude that

$$
G\left(u_{n}, u_{n}, u_{n+2}\right) \leq \varphi^{n}\left(G\left(u_{0}, u_{0}, u_{2}\right)\right) .
$$

Therefore, we obtain

$$
\lim _{n \rightarrow \infty} G\left(u_{n}, u_{n}, u_{n+2}\right)=\lim _{n \rightarrow \infty} G\left(u_{n}, u_{n+2}, u_{n+2}\right)=0 .
$$

Next, we will show that $\left\{u_{n}\right\}$ is a $G$-Cauchy sequence in $E$ by contradiction. If $\left\{u_{n}\right\}$ is not a $G$-Cauchy sequence, then there exists $\varepsilon>0$ and the subsequences $u_{n}(k), u_{m(k)}$ and $u_{l(k)}$ of $\left\{u_{n}\right\}$ such for $n(k)>m(k)>l(k)>k$ with $G\left(u_{n(k)}, u_{m(k)}, u_{l(k)}\right) \geq \varepsilon$ and $G\left(u_{n(k)-1}, u_{m(k)}, u_{l(k)}\right)<\varepsilon$. On the one hand,

$$
\begin{aligned}
\varepsilon \leq & G\left(u_{n(k)}, u_{m(k)}, u_{l(k)}\right) \\
\leq s & {\left[G\left(u_{m(k)}, u_{m(k)+2}, u_{m(k)+2}\right)+G\left(u_{m(k)+2}, u_{m(k)+1}, u_{m(k)+1}\right)\right.} \\
& \left.+G\left(u_{m(k)+1}, u_{n(k)}, u_{l(k)}\right)\right] \\
\leq & s\left[G\left(u_{m(k)}, u_{m(k)+2}, u_{m(k)+2}\right)+G\left(u_{m(k)+2}, u_{m(k)+1}, u_{m(k)+1}\right)\right] \\
& +s^{2}\left[G\left(u_{l(k)}, u_{l(k)+2}, u_{l(k)+2}\right)+G\left(u_{l(k)+2}, u_{l(k)+1}, u_{l(k)+1}\right)+G\left(u_{l(k)+1}, u_{m(k)+1}, u_{n(k)}\right)\right] \\
\leq & s\left[G\left(u_{m(k)}, u_{m(k)+2}, u_{m(k)+3}\right)+G\left(u_{m(k)+2}, u_{m(k)+1}, u_{m(k)}\right)\right]+s^{2}\left[G\left(u_{l(k)}, u_{l(k)+1}, u_{l(k)+2}\right)\right. \\
& \left.+G\left(u_{l(k)+2}, u_{l(k)+1}, u_{l(k)}\right)+G\left(u_{l(k)+1}, u_{m(k)+1}, u_{n(k)}\right)\right]
\end{aligned}
$$

taking the limits as $k \rightarrow \infty$ in above inequality, we conclude

$$
\frac{\varepsilon}{s^{2}} \leq \lim _{k \rightarrow \infty} G\left(u_{l(k)+1}, u_{m(k)+1}, u_{n(k)}\right) \text {. }
$$

On the other hand,

$$
\begin{aligned}
G\left(u_{l(k)+1}, u_{m(k)+1}, u_{n(k)}\right) & =G\left(T_{\alpha_{l(k)}} u_{l(k)}, T_{\alpha_{m(k)}} u_{m(k)}, T_{\alpha_{n(k)-1}} u_{n(k)-1}\right) \\
& \leq \frac{1}{s^{2}} \varphi\left(G\left(u_{l(k)}, u_{m(k)}, u_{n(k)-1}\right)\right),
\end{aligned}
$$

let $k \rightarrow \infty$ in above inequality, we obtain

$$
\frac{\varepsilon}{s^{2}} \leq \frac{1}{s^{2}} \varphi(\varepsilon)<\frac{\varepsilon}{s^{2}}
$$

a contradiction. Thus, $\left\{u_{n}\right\}$ is a $G-$ Cauchy sequence. By completeness of $(E, d, w)$, there exists $u^{*} \in E$ such that $\lim _{n \rightarrow \infty} u_{n}=u^{*}$. Let us show that $u^{*}$ is a fixed point of $T_{\alpha}$. Applying the rectangular inequality, we obtain that

$$
\begin{aligned}
G\left(u^{*}, T_{\alpha} u^{*}, T_{\alpha} u^{*}\right) & \leq s\left[G\left(u^{*}, u_{n}, u_{n}\right)+G\left(u_{n}, u_{n+1}, u_{n+1}\right)+G\left(u_{n+1}, T_{\alpha} u^{*}, T_{\alpha} u^{*}\right)\right] \\
& =s\left[G\left(u^{*}, u_{n}, u_{n}\right)+G\left(u_{n}, u_{n+1}, u_{n+1}\right)+G\left(T_{\alpha} u_{n}, T_{\alpha} u^{*}, T_{\alpha} u^{*}\right)\right] \\
& \leq s\left[G\left(u^{*}, u_{n}, u_{n}\right)+G\left(u_{n}, u_{n+1}, u_{n+1}\right)+\frac{1}{s^{2}} \varphi\left(G\left(u_{n}, u^{*}, u^{*}\right)\right)\right],
\end{aligned}
$$

letting $n \rightarrow \infty$, we deduce $G\left(u^{*}, T_{\alpha} u^{*}, T_{\alpha} u^{*}\right)=0$ which implies $u^{*} \in F\left(T_{\alpha}\right)$. Suppose that $u^{*}, v^{*} \in E$ are two distinct fixed points of $T_{\alpha}$. That is, $G\left(u^{*}, u^{*}, v^{*}\right)>0$. Then

$$
G\left(u^{*}, u^{*}, v^{*}\right)=G\left(T_{\alpha} u^{*}, T_{\alpha} u^{*}, T_{\alpha} v^{*}\right) \leq \frac{1}{s^{2}} G\left(u^{*}, u^{*}, v^{*}\right)<G\left(u^{*}, u^{*}, v^{*}\right),
$$


which is a contradiction. Therefore, we must have $G\left(u^{*}, u^{*}, v^{*}\right)=0$, that is, $u^{*}=v^{*}$. Thus $T_{\alpha}$ has a unique fixed point in $E$. To finish the proof, we apply Lemma 4.

Taking $\varphi(t)=\lambda t$ in Theorem 4 , we obtain the following result.

Corollary 3. Let $(E, G, w)$ be a $C R G_{b} M S$ with $s \geq 1$ and let $T: E \rightarrow E$ be an enriched contraction or $(\alpha, \lambda)$-enriched contraction, that is, there exists $\alpha \in[0,1)$ and $\lambda \in\left[0, \frac{1}{s^{2}}\right)$ such that

$$
G(w(u, T u ; \alpha), w(v, T v ; \alpha), w(o, T o ; \alpha)) \leq \lambda G(u, v, o)
$$

for all $u, v, o \in E$. Then,

(1) $F(T)=\left\{u^{*}\right\}$, for some $u^{*} \in E$;

(2) the sequence $\left\{u_{n}\right\}$ obtained from the iterative process

$$
u_{n+1}=w\left(u_{n}, T u_{n} ; \alpha_{n}\right)
$$

converges to $u^{*}$, for any $u_{0} \in E$.

The result of the local variant of enriched contractions in $C R G_{b} M S$ as following.

Theorem 5. Let $(E, G, w)$ be a $C R G_{b} M S$ with $s \geq 1$ and $B=B_{G}\left(u_{0}, r\right), u_{0} \in E, r>0$. Assume that $T: B \rightarrow E$ be an enriched contraction and $0<\alpha<1$. If

$$
\max \left\{G\left(u_{0}, u_{0}, T u_{0}\right), G\left(u_{0}, T u_{0}, T u_{0}\right)\right\} \leq\left(\frac{1}{2 s}-\frac{\lambda}{2}\right) \varepsilon,
$$

then $T$ has a fixed point in $E$.

Proof. We can choose $\varepsilon<r$ such that

$$
\max \left\{G\left(u_{0}, u_{0}, T u_{0}\right), G\left(u_{0}, T u_{0}, T u_{0}\right)\right\} \leq\left(\frac{1}{2 s}-\frac{\lambda}{2}\right) \varepsilon<\left(\frac{1}{2 s}-\frac{\lambda}{2}\right) r .
$$

Since $T$ is a enriched contraction, for all $u, v, o \in E$ and any $\alpha \in[0,1)$, we can apply the contractive condition (10) with $T_{\alpha} u=w(u, T u ; \alpha)$, then we obtain

$$
G\left(T_{\alpha} u, T_{\alpha} v, T_{\alpha} o\right) \leq \lambda G(u, v, o) .
$$

We shall prove that the closed ball

$$
\bar{B}_{G}\left(u_{0}, \varepsilon\right)=\left\{u \in E: G\left(u_{0}, u, u\right) \leq \varepsilon\right\}
$$

is invariant with respect to $T_{\alpha}$. Suppose that $u_{0}$ is not a fixed point of $T$, that is, $u_{0} \neq T u_{0}$ and $u_{0} \neq T_{\alpha} u_{0}$. Due to $\alpha>0$, it is not difficult to see that $T u_{0} \neq T_{\alpha} u_{0}$, indeed, if not,

$$
\begin{aligned}
G\left(u_{0}, u_{0}, T u_{0}\right) & =G\left(u_{0}, u_{0}, T_{\alpha} u_{0}\right) \\
& =G\left(u_{0}, u_{0}, w\left(u_{0}, T u_{0} ; \alpha\right)\right) \\
& \leq(1-\alpha)\left(u_{0}, u_{0}, T u_{0}\right),
\end{aligned}
$$

a contradiction. For any $u \in \bar{B}_{G}\left(u_{0}, \varepsilon\right)$, we will consider the following three cases: 
Case 1: if $T u_{0}=T_{\alpha} u$, we have

$$
\begin{aligned}
G\left(u_{0}, T_{\alpha} u, T_{\alpha} u\right) & =G\left(u_{0}, T u_{0}, T u_{0}\right) \\
& \leq G\left(u_{0}, T u_{0}, T_{\alpha} u_{0}\right) \\
& =G\left(u_{0}, T u_{0}, w\left(u_{0}, T u_{0} ; \alpha\right)\right) \\
& \leq \alpha G\left(u_{0}, u_{0}, T u_{0}\right)+(1-\alpha) G\left(u_{0}, T u_{0}, T u_{0}\right) \\
& \leq\left(\frac{1}{2 s}-\frac{\lambda}{2}\right) \varepsilon<\varepsilon ;
\end{aligned}
$$

Case 2: if $T_{\alpha} u_{0}=T_{\alpha} u$, we have

$$
\begin{aligned}
G\left(u_{0}, T_{\alpha} u, T_{\alpha} u\right) & =G\left(u_{0}, T_{\alpha} u_{0}, T_{\alpha} u_{0}\right) \\
& \leq G\left(u_{0}, T u_{0}, T_{\alpha} u_{0}\right) \\
& =G\left(u_{0}, T u_{0}, w\left(u_{0}, T u_{0} ; \alpha\right)\right) \\
& \leq \alpha G\left(u_{0}, u_{0}, T u_{0}\right)+(1-\alpha) G\left(u_{0}, T u_{0}, T u_{0}\right) \\
& \leq\left(\frac{1}{2 s}-\frac{\lambda}{2}\right) \varepsilon<\varepsilon ;
\end{aligned}
$$

Case 3: if $T u_{0} \neq T_{\alpha} u$ and $T_{\alpha} u_{0} \neq T_{\alpha} u$, we have

$$
\begin{aligned}
G\left(u_{0}, T_{\alpha} u, T_{\alpha} u\right) \leq & s\left[G\left(u_{0}, T u_{0}, T u_{0}\right)+G\left(T u_{0}, T_{\alpha} u_{0}, T_{\alpha} u_{0}\right)+G\left(T_{\alpha} u_{0}, T_{\alpha} u, T_{\alpha} u\right)\right] \\
\leq & s\left[G\left(u_{0}, T u_{0}, T u_{0}\right)+G\left(T u_{0}, u_{0}, T_{\alpha} u_{0}\right)+G\left(T_{\alpha} u_{0}, T_{\alpha} u, T_{\alpha} u\right)\right] \\
= & s\left[G\left(u_{0}, T u_{0}, T u_{0}\right)+G\left(T u_{0}, u_{0}, w\left(u_{0}, T u_{0}, \alpha\right)\right)+G\left(T_{\alpha} u_{0}, T_{\alpha} u, T_{\alpha} u\right)\right] \\
\leq & s\left[G\left(u_{0}, T u_{0}, T u_{0}\right)+\alpha G\left(T u_{0}, u_{0}, u_{0}\right)+(1-\alpha) G\left(T u_{0}, u_{0}, T u_{0}\right)\right. \\
& \left.+\lambda G\left(u_{0}, u, u\right)\right] \\
\leq & s\left[\left(\frac{1}{s}-\lambda\right) \varepsilon+\lambda \varepsilon\right]=\varepsilon .
\end{aligned}
$$

Finally, by above cases, we prove that $G\left(u_{0}, T_{\alpha} u, T_{\alpha} u\right) \leq \varepsilon$, which implies $T_{\alpha} u \in$ $\bar{B}_{G}\left(u_{0}, \varepsilon\right)$. Since $\bar{B}_{G}\left(u_{0}, \varepsilon\right)$ is complete, the result follows from Corollary 3 .

Author Contributions: C.L. and Y.C. contributed equally and significantly in writing this article. All authors have read and agreed to the published version of the manuscript.

Funding: This work was supported by the National Natural Science Foundation of China under Grants 11871181.

Institutional Review Board Statement: Not applicable.

Informed Consent Statement: Not applicable.

Data Availability Statement: Not applicable.

Acknowledgments: We would like to express our thanks to the anonymous referees and the editor for their constructive comments and suggestions, which greatly improved this article.

Conflicts of Interest: The authors declare no conflict of interest.

\section{References}

1. Czerwik, S. Contraction mappings in b-metric spaces. Acta Math. Inform. Univ. Ostrav. 1993, 1, 5-11.

2. Banach, S. Sur les operations dans les ensembles abstraits et leur application aux equations integrales. Fund. Math. 1922, 3, 133-181. [CrossRef]

3. Branciari, A. A fixed point theorem of Banach-Caccioppoli type on a class of generalized metric spaces. Publ. Math. 2000, 57, 31-37.

4. George, R.; Radenovi, S.; Reshma, K.P.; Shukla, S. Rectangular b-metric space and contraction principles J. Nonlinear Sci. Appl. 2015, 8, 1005-1013. [CrossRef]

5. Mustafa, Z.; Sims, B. A new approach to generalized metric spaces. J. Nonlinear Convex A 2006, 7, $289-297$. 
6. Mustafa, Z. A New Structure for Generalized Metric Spaces: With Applications to Fixed Point Theory. Ph.D. Thesis, University of Newcastle, Newcastle, UK, 2005.

7. Aghajani, A.; Abbas, M.; Roshan, J. Common fixed point of generalized weak contractive mappings in partially ordered $b$-metric spaces. Math. Slovaca 2014, 4, 941-960. [CrossRef]

8. Aydi, H.; Rakić, D.; Aghajani, A.; Došenović, T.; Noorani, M.S.M.; Qawaqneh, H. On fixed point results in $G_{b}$-metric spaces. Mathematics 2019, 7, 617. [CrossRef]

9. Agarwal, R.P.; Karapınar, E.; O’Regan, D.; Roldán-López-de-Hierro, A.F. Fixed Point Theory in Metric Type Spaces; Springer International Publishing: Gewerbestrasse, Switzerland, 2015.

10. Roldán-López-de-Hierro, A.F.; Karapınar, E.; O’Regan, D.; Karapinar, E. Coincidence point theorems on quasi-metric spaces via simulation functions and applications to G-metric spaces. J. Fixed Point Theory Appl. 2018, 20,1661-7738. [CrossRef]

11. Reich, S. Fixed point theory in locally convex spaces. Math. Z. 1972, 125, 17-31. [CrossRef]

12. Reich, S.; Shafrir, I. Nonexpansive iterations in hyperbolic spaces. Nonlinear Anal. 1990, 19, 537-558. [CrossRef]

13. Chen, L.; Gao, L.; Zhao, Y. A new iterative scheme for finding attractive points of $(\alpha, \beta)$-generalized hybrid set-valued mappings. J. Nonlinear Sci. Appl. 2017, 10, 1228-1237. [CrossRef]

14. Chen, L.; Gao, L.; Chen, D. Fixed point theorems of mean nonexpansive set-valued mappings in Banach spaces. J. Fixed Point Theory Appl. 2017, 19, 2129-2143. [CrossRef]

15. Chen, L.; Yang, N.; Zhao, Y.; Ma, Z. Fixed point theorems for set-valued G-contractions in a graphical convex metric space with applications. Discret. Contin. Dyn. Syst. 2020, 22, 88. [CrossRef]

16. Chen, L.; Huang, S.; Li, C.; Zhao, Y. Several fixed point theorems for F-contractions in complete Branciari $b$-metric spaces and applications. J. Funct. Space. 2020, 69, 1-10. [CrossRef]

17. Karapınar, E.; Chifu, E. Results in wt-Distance over b-metric Spaces. Mathematics 2020, 8, 220. [CrossRef]

18. Chifu, C.; Karapınar, E.; Petrusel, P. Fixed Point Results for Frum-Ketkov Type Contractions in b-metric Spaces. Axioms 2021, 10, 231. [CrossRef]

19. Takahashi, W. A convexity in metric space and nonexpansive mappings I. Kodai Math J. 1970, 22, 142-149. [CrossRef]

20. Chen, L.; Li, C.; Kaczmarek, R.; Zhao, Y. Several fixed point theorems in convex $b$-metric spaces and applications. Mathematics 2020, 8, 242. [CrossRef]

21. Berinde, V.; Păcurar, M. Approximating fixed points of enriched contractions in Banach spaces. J. Fixed Point Theory Appl. 2020, 22, 1-10. [CrossRef]

22. Berinde, V.; Păcurar, M. Kannan's fixed point approximation for solving split feasibility and variational inequality problems. J. Comput. Appl. Math. 2021, 386, 113217. [CrossRef]

23. Berinde, V.; Păcurar, M. Fixed points tTheorems for unsaturated and saturated classes of contractive mappings in Banach spaces. Symmetry 2021, 13, 713. [CrossRef]

24. Abbas, M.; Anjum, R.; Berinde, V. Enriched multivalued contractions with applications to differential inclusions and dynamic programming. Symmetry 2021, 13, 1350. [CrossRef]

25. Berinde, V.; Păcurar, M. Existence and approximation of fixed Points of enriched contractions and enriched $\varphi$-contractions. Symmetry 2021, 13, 498. [CrossRef]

26. Mitrović, Z.D. On an open problem in rectangular b-metric space. J. Anal. 2017, 25, 135-137. [CrossRef]

27. Browder, F.E.; Petryshyn, W.V. Construction of fixed points of nonlinear mappings in Hilbert space. J. Math. Anal. Appl. 1967, 20, 197-228. [CrossRef]

28. Berinde, V. Generalized Contractions and Applications; Editura Cub Press: Baia Mare, Romania, 1997. 